A Nitsche Embedded Mesh Method

J. D. Sanders, T. A. Laursen, M. A. Puso

February 23, 2011

Computational Mechanics 
This document was prepared as an account of work sponsored by an agency of the United States government. Neither the United States government nor Lawrence Livermore National Security, LLC, nor any of their employees makes any warranty, expressed or implied, or assumes any legal liability or responsibility for the accuracy, completeness, or usefulness of any information, apparatus, product, or process disclosed, or represents that its use would not infringe privately owned rights. Reference herein to any specific commercial product, process, or service by trade name, trademark, manufacturer, or otherwise does not necessarily constitute or imply its endorsement, recommendation, or favoring by the United States government or Lawrence Livermore National Security, LLC. The views and opinions of authors expressed herein do not necessarily state or reflect those of the United States government or Lawrence Livermore National Security, LLC, and shall not be used for advertising or product endorsement purposes. 


\title{
A Nitsche Embedded Mesh Method
}

\author{
Jessica Sanders ${ }^{1, *}$, Tod A. Laursen ${ }^{2}$, Michael A. Puso ${ }^{1}$
}

\begin{abstract}
A new technique for treating the mechanical interactions of overlapping finite element meshes is proposed. Numerous names have been applied to related approaches, here we refer to such techniques as embedded mesh methods. Such methods are useful for numerous applications e.g., fluid-solid interaction with a superposed meshed solid on an Eulerian background fluid grid or solid-solid interaction with a superposed meshed particle on a matrix background mesh etc. In this work we consider the interaction of two elastic domains: one mesh is the foreground and defines the surface of interaction, the other is a background mesh and is often a grid. We first employ a classical mortar type approach (see [1]) to impose constraints on the interface. It turns out that this approach will work well except in special cases. In fact, many related approaches can exhibit mesh locking under certain conditions. This motivates the proposed version of Nitsche's method which is shown to eliminate the locking phenomenon in example problems.
\end{abstract}

Keywords: embedded mesh, mortar method, Nitsche's method, interfaces

\section{Introduction}

In this paper, we consider the important problem of imposing continuity restrictions at the junction of overlapping finite element grids, as depicted in Figure 1. A vast amount of literature exists on such approaches, ranging from fictitious domain methods (FD), which use Lagrange multipliers to enforce continuity between a body or obstacle and a background grid (see $[2,1]$ ); to a variety of over-set grid methods (see $[3,4,5]$ ) which to a large extent follow ideas going back at least to Noh 1964 (see [6]); to the Arlequin method (see [7]); to the rapidly growing literature on how interface constraints and constitutive laws can be imposed within the context of extended finite element methods and other related approaches (see $[8,9,10,11])$.

In our applications of overlapping finite element grids, we seek to demonstrate and address the issue of "mesh locking" which may occur when the number of constraints imposed

\footnotetext{
${ }^{*}$ Corresponding author. Address: 7000 East Ave, Livermore, CA , 94550

Email addresses: sanders39@llnl.gov (Jessica Sanders), tod.laursen@kustar.ac.ae (Tod A. Laursen), puso1@llnl.gov (Michael A. Puso)

${ }^{1}$ Methods Development Group, Lawrence Livermore National Laboratory, Livermore, CA 94550

${ }^{2}$ Khalifa University of Science, Technology \& Research, Abu Dhabi, UAE
} 


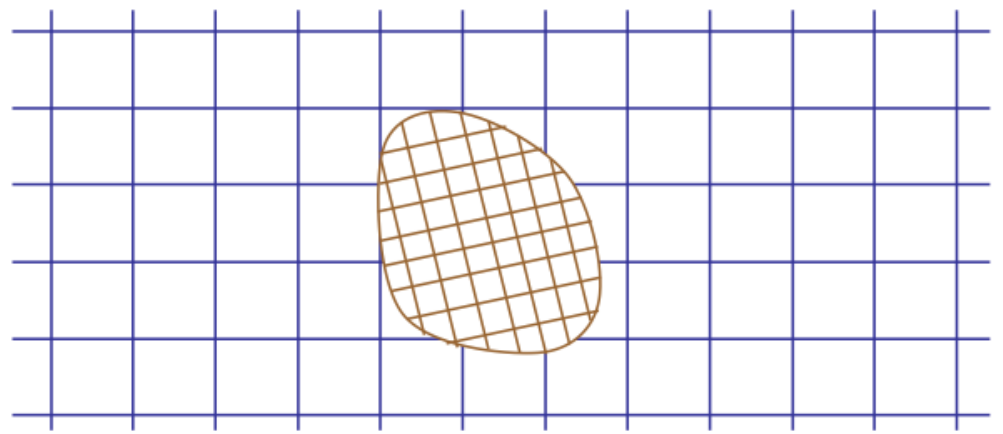

Figure 1: Finite element embedded mesh approach: A finely gridded Lagrangian body overlaps a coarser grid representing either an Eulerian or Lagrangian background material.

on the surface or volume exceeds the number of degrees of freedom available to render these constraints independent of the physical deformations the elements should allow. This generally occurs when a domain with a very fine mesh overlays a coarser background grid. Oscillations of the Lagrange multipliers (when they are used to impose the constraints) may also accompany this situation. This problem manifests itself in both loss of overall accuracy and suboptimal convergence rates in affected problems. On the other hand, a relative scarcity of constraints can lead to under-representation of the compatibility conditions and inaccurate representations of surface fluxes. An additional challenge in solving these problems is that we generally wish that low-order approximations (typically linear velocities or displacements, spatially constant fluxes) be exactly representable across tied overlap regions (at the very least for flat interfaces, and ideally more broadly).

Many, if not most, of these challenges have been met within the contexts of the aforementioned methods. For this work, however, we ask the question whether such requirements can be met under rather specific circumstances. Specifically, our goals and assumptions are:

1. We are most interested in avoidance of mesh locking (as manifested, for example, by lack of optimality in bulk $L_{2}$ and $H_{1}$ norms of error).

2. The method should be applicable in either of two or three spatial dimensions (as is usual in computational mechanics, the latter case is much more difficult in general)

3. Conservation of linear momentum (for all geometries) is an important feature we wish to maintain, as is passage of patch tests (at least for flat interfaces, but preferably curved as well).

4. We wish to employ a framework in which we enforce surface to volume constraints, as opposed to the more commonly applied surface-to-surface or volume-to-volume constraints treated in contact.

It should be mentioned that items 2 and 3 are particularly demanding, especially in light of recent efforts in the literature. For example, Bechet, Moes and Wohlmuth (see [9]) propose a particularly clever way of sorting through nodes and constraints in the context 
of an enriched interface method, identifying so-called "vital nodes" that ensure a stable multiplier representation through use also of the trace of the element shape functions as a multiplier basis. The resulting method is convincingly demonstrated to satisfy inf-sup, though application of the method is involved and possibly inconvenient. It has not, to the authors' knowledge, been applied to the problem of overlapping domains although its extension would be natural.

Here we begin with a rather standard mortar method to join the surface of one elastic domain to the image of this surface in an overlapped second elastic domain. Though the method is broadly applicable in many cases, this method displays mesh locking in the case where the overlapping mesh is both finer and stiffer than the underlying grid, as will be demonstrated in our numerical examples. A penalty approximation is also studied, and shown to exhibit sub-optimal convergence rates due to loss of consistency. We finally study Nitsche's method as an alternative approach to applying the continuity constraints. Nitsche's method has already been studied for overlapping domains for the Laplace equation in [12]), though the issue of mesh locking is not investigated, probably due to the equivalent stiffness of the domains. In this case, we uncover the surprising results that for the same pathological situation in which standard application of mortar constraints produces mesh locking, the typical application of Nitsche's method also produces mesh locking and sub-par convergence behavior. Here we show that a weighted method biased to the background grid alleviates locking and provides convergence of the relevant norms.

This document is organized as follows. Section 2 introduces the problem formulation and governing equations for the embedded mesh formulation in a general sense, for both linear and non-linear problems. Sections 3 and 4 describe a straight forward implementation of the mortar approach, and a penalty approximation, repectively. Section 5 describes the application of Nitsche's method to a linear form of the problem, including a derivation of the weighted form. Section 6 overviews the global equation structure produced by the procedure, while Section 7 provides some numerical demonstrations of the technique.

\section{Problem Formulation}

We consider, as a model problem, the perfect adhesion of two linear elastic bodies in a static framework. As depicted in Figure 2, we assume that one body, $B^{1}$, overlays the second body, $B^{2}$, over some overlap region, and we denote the intersection of $\partial B^{1}$ (the boundary of $B^{1}$ ) with $B^{2}$ as $\Gamma_{*}$. In what follows, the coordinates in bodies $B^{i}, i=1,2$, will be denoted as $\boldsymbol{x}^{1}$ and $\boldsymbol{x}^{2}$, respectively. The entire computational domain is considered as the union of $B^{1}$, with the uncovered region of $B^{2}, \Omega=B^{1} \cup\left(B^{2} \backslash B^{1}\right)$. Our formulation will be general to cases of both complete (as shown) and partial overlap of the bodies. Excluding $\Gamma_{*}$, the rest of the computational boundary can be divided into Dirichlet and Neumann parts on each

body, $\Gamma_{d}^{(m)}$ and $\Gamma_{h}^{(m)}$ respectively. A super script, $(m)$, is used to denote a quantity that is valid over region $B^{m}$, with $m=1,2$.

The system of linear elastostatic equilibrium equations with the primary unknown dis- 


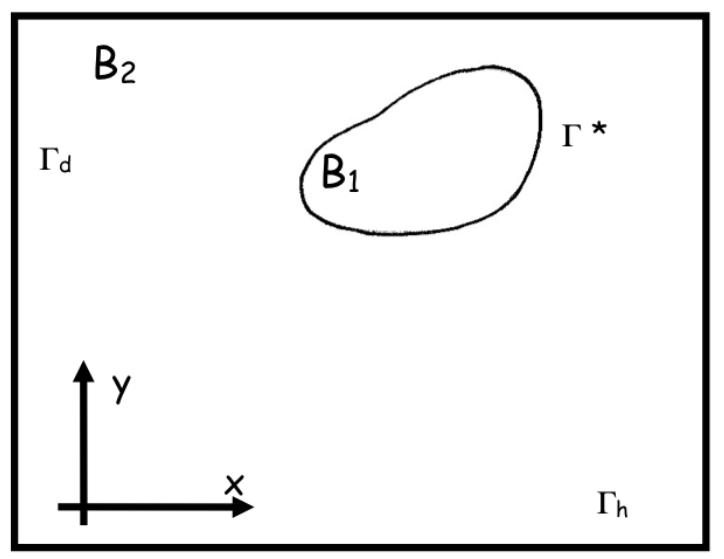

Figure 2: Two body problem domain

placement, $\boldsymbol{u}^{(m)}$, completed with boundary conditions, is

$$
\begin{aligned}
\nabla \boldsymbol{\sigma}^{(m)}+\boldsymbol{f}^{(m)} & =0 & & \text { in } \Omega \\
\boldsymbol{u}^{(m)} & =\overline{\boldsymbol{u}}^{(m)} & & \text { on } \Gamma_{d}^{(m)} \\
\boldsymbol{\sigma}^{(m)} \boldsymbol{n}^{(m)} & =\boldsymbol{h}^{(m)} & & \text { on } \Gamma_{h}^{(m)} \\
\boldsymbol{u}^{(1)} & =\boldsymbol{u}^{(2)} & & \text { on } \Gamma_{*} \\
\boldsymbol{\sigma}^{(1)} \boldsymbol{n}^{(1)} & =-\boldsymbol{\sigma}^{(2)} \boldsymbol{n}^{(2)} & & \text { on } \Gamma_{*}
\end{aligned}
$$

where the strain is taken as the symmetric part of the displacement gradient:

$$
\boldsymbol{\varepsilon}(\boldsymbol{u})^{(m)}=\frac{1}{2}\left(\nabla \boldsymbol{u}^{(m)}+\nabla^{T} \boldsymbol{u}^{(m)}\right)
$$

and the Cauchy stress is a linear function of the strains:

$$
\boldsymbol{\sigma}^{(m)}=\boldsymbol{C}^{(m)}: \boldsymbol{\varepsilon}^{(m)}(\boldsymbol{u})
$$

Here, $\boldsymbol{C}^{(m)}$ is the fourth order tensor of material properties for body $m$ according to Hooke's Law.

\section{Mortar Tying of a Surface to an Overlapped Volume}

We start by defining the spaces, $\boldsymbol{S}$ and $\boldsymbol{V}$, defined over each subdomain, $B^{1}$ and $B^{2}$, that will contain the solution and trial functions respectively:

$$
\begin{gathered}
\boldsymbol{S}^{(m)}=\left\{\boldsymbol{u}(\boldsymbol{x}) \mid \boldsymbol{u}(\boldsymbol{x}) \in \boldsymbol{H}^{1}\left(B^{m}\right), \boldsymbol{u}(\boldsymbol{x})=\overline{\boldsymbol{u}}(\boldsymbol{x}) \text { on } \Gamma_{d}\right\} \\
\boldsymbol{V}^{(m)}=\left\{\boldsymbol{w}(\boldsymbol{x}) \mid \boldsymbol{w}(\boldsymbol{x}) \in \boldsymbol{H}^{1}\left(B^{m}\right), \boldsymbol{w}(\boldsymbol{x})=\mathbf{0} \text { on } \Gamma_{d}\right\}
\end{gathered}
$$


It is important to note that while these functions are continuous within each subdomain, the restriction of the continuity to individual bodies means the entire solution may be discontinuous at the boundary $\Gamma_{*}$. The mortar approach, used for example by [1], would (weakly) constrain the surface of $B^{1}$ to move in concert with the underlying mesh of $B^{2}$ via a mixed variational form. We choose to enforce the constraints (1d) and (1e) via a traction field, belonging to space $\boldsymbol{L}$, represented by $\boldsymbol{\lambda}$ acting over the interface $\Gamma_{*}$,

$$
\boldsymbol{L}=\left\{\boldsymbol{\lambda}(\boldsymbol{x}) \mid \boldsymbol{\lambda}(\boldsymbol{x}) \in L^{2}\left(\Gamma_{*}\right)\right\}
$$

The mixed variational statement for this method is:

Find $\left(\boldsymbol{u}^{(1)}, \boldsymbol{u}^{(2)}, \boldsymbol{\lambda}\right) \in \boldsymbol{S}^{(1)} \times \boldsymbol{S}^{(2)} \times \boldsymbol{L}$ such that

$$
\begin{aligned}
\sum_{m=1}^{2} \int_{B^{m}} \boldsymbol{\varepsilon}(\boldsymbol{w})^{(m)}: \boldsymbol{\sigma}^{(m)} d B+\int_{\Gamma_{*}} \boldsymbol{\lambda} \cdot[[\boldsymbol{w}]] d \Gamma= \\
\sum_{m=1}^{2} \int_{B^{m}} \boldsymbol{w}^{(m)} \cdot \boldsymbol{f}^{(m)} d B+\sum_{m=1}^{2} \int_{\Gamma_{h}^{(m)}} \boldsymbol{w}^{(m)} \cdot \boldsymbol{h}^{(m)} d \Gamma,
\end{aligned}
$$

and

$$
\int_{\Gamma_{*}} \boldsymbol{\mu} \cdot[[\boldsymbol{u}]] d \Gamma=0
$$

for all $\left(\boldsymbol{w}^{(1)}, \boldsymbol{w}^{(2)}, \boldsymbol{\mu}\right) \in \boldsymbol{V}^{(1)} \times \boldsymbol{V}^{(2)} \times \boldsymbol{L}$.

A gap function is defined on $\Gamma_{*}$ as $[[\boldsymbol{u}]]=\boldsymbol{u}^{1}-\boldsymbol{u}^{2}$. Equation (2) is a statement of conservation of momentum for the entire system. The "constraint" virtual work,

$$
G^{C}=\int_{\Gamma_{*}} \boldsymbol{\lambda} \cdot\left(\boldsymbol{w}^{1}-\boldsymbol{w}^{2}\right) d \Gamma_{*},
$$

represents the additional internal work the system performs in order to maintain a zero gap function. Equation (3) is a constraint equation enforcing that the gap must vanish in a weak, or integral, sense.

A major disadvantage of mixed methods (called mixed because they solve simultaneously for two different fields, in this case displacement and traction) is that when discretized, their performance is dependent on the finite element subspaces satisfying the so-called LBB (or inf-sup) conditions. For embedded interface methods, this rules out many convenient choices for the subspaces. The various problems with getting stable and convergent results will be demonstrated in Section 7. 


\section{Penalty Approximations to Mortar Lagrange Multipliers}

One way of avoiding a mixed variational formulation would be to apply a penalty approximation of the mortar constraints. Instead of explicitly solving for the Lagrange multiplier field, we approximate it with

$$
\boldsymbol{\lambda} \approx \frac{\alpha}{2}[[\boldsymbol{u}]]
$$

where the scalar constant $\alpha$ can be interpreted as a gap stiffness, and will be referred to as the penalty parameter. The associated variational form is:

Find $\left(\boldsymbol{u}^{(1)}, \boldsymbol{u}^{(2)}\right) \in \boldsymbol{S}^{(1)} \times \boldsymbol{S}^{(2)}$ such that

$$
\begin{aligned}
\sum_{m=1}^{2} \int_{B^{m}} \boldsymbol{\varepsilon}(\boldsymbol{w})^{(m)}: & \boldsymbol{\sigma}^{(m)} d B+\alpha \int_{\Gamma_{*}}[[(\boldsymbol{u})]] \cdot[[(\boldsymbol{w})]] d \Gamma= \\
& \sum_{m=1}^{2} \int_{B^{m}} \boldsymbol{w}^{(m)} \cdot \boldsymbol{f}^{(m)} d B+\sum_{m=1}^{2} \int_{\Gamma_{h}^{(m)}} \boldsymbol{w}^{(m)} \cdot \boldsymbol{h}^{(m)} d \Gamma \\
& \text { for all }\left(\boldsymbol{w}^{(1)}, \boldsymbol{w}^{(2)}\right) \in \boldsymbol{V}^{(1)} \times \boldsymbol{V}^{(2)} .
\end{aligned}
$$

The advantage of the penalty method is that there are no additional global constraint equations and only one unknown field (displacement). However, the penalty method is variationally inconsistent, and discrete results are overly sensitive to $\alpha$. A classic result by [13] indicates that to achieve spatial convergence, the penalty parameter must grow at a rate that outpaces the other terms in the stiffness matrix. Additionally, the constraint is only perfectly represented in the limit $\alpha \rightarrow \infty$. For the discretized method, this eventually leads to poor conditioning of the linear algebraic system of equations.

\section{Nitsche's Method}

Unfortunately, given the way we choose our constraints in the discrete form of the standard mortar method, we find ourselves subject to the widely identified problem of mesh locking (see, for example, thorough descriptions and examples in [9] or [14]). The logical choice in this case is to define the basis for the discrete Lagrange multipliers mesh on the surface $\Gamma_{*}$. Within the context of our formulation, the problem occurs on the master side (body 2), where the interface is effectively embedded with the volume. Particularly for the case where the discretization of the slave side is comparatively fine relative to the master side volume discretization, use of the standard mortar approximations will give rise to increasingly severe over-constraint as the mesh is refined. A possible way of circumventing these problems is to apply a weak constraint enforcement based on Nitsche's method. The standard application of Nitsche's method for the two-body problem is: 
Find $\left(\boldsymbol{u}^{(1)}, \boldsymbol{u}^{(2)}\right) \in \boldsymbol{S}^{(1)} \times \boldsymbol{S}^{(2)}$ such that

$$
\begin{aligned}
& \sum_{m}^{2} \int_{B^{m}} \boldsymbol{\varepsilon}(\boldsymbol{w})^{(m)}: \boldsymbol{\sigma}^{(m)} d B-\int_{\Gamma_{*}}\left([[\boldsymbol{w}]] \otimes \boldsymbol{n}^{(1)}\right):\langle\boldsymbol{\sigma}\rangle d \Gamma \\
&- \int_{\Gamma_{*}}\left([[\boldsymbol{u}]] \otimes \boldsymbol{n}^{(1)}\right):\langle\boldsymbol{\sigma}(\boldsymbol{w})\rangle d \Gamma+\beta \int_{\Gamma_{*}}[[\boldsymbol{u}]] \cdot[[\boldsymbol{w}]] d \Gamma= \\
& \sum_{m=1}^{2} \int_{B^{m}} \boldsymbol{w}^{(m)} \cdot \boldsymbol{f}^{(m)} d B+\sum_{m=1}^{2} \int_{\Gamma_{h}^{(m)}} \boldsymbol{w}^{(m)} \cdot \boldsymbol{h}^{(m)} d \Gamma \\
& \text { for all }\left(\boldsymbol{w}^{(1)}, \boldsymbol{w}^{(2)}\right) \in \boldsymbol{V}^{(1)} \times \boldsymbol{V}^{(2)}
\end{aligned}
$$

Here the brackets \langle\rangle indicate an average quantity, $\langle a\rangle=\frac{1}{2}\left(a^{(1)}+a^{(2)}\right)$, and for linear elasticity the operator $\boldsymbol{\sigma}(\boldsymbol{w})$ is defined:

$$
\boldsymbol{\sigma}(\boldsymbol{w})=\boldsymbol{C}: \boldsymbol{\varepsilon}(\boldsymbol{w})
$$

With the exception of the second and third terms on the left side, the equilibrium condition is the same as that for the penalty method. Nitsche's method assures continuity of the solution vector at $\Gamma_{*}$ by directly correcting a consistency error that arises due to mismatching weighting functions at the interface. That correction comes from the extra terms not present in a penalty formulation.

Nitsche's method can be easily derived from a stored energy functional approach, as follows: The total potential energy of the two body system can be computed as the difference between energy due to internal and external forces:

$$
\Pi(\boldsymbol{u})=\frac{1}{2} \sum_{m=1}^{2} \int_{B^{m}} \boldsymbol{\varepsilon}^{(m)}: \boldsymbol{\sigma}^{(m)} d B-\sum_{m=1}^{2} \int_{\Gamma_{h}^{(m)}} \boldsymbol{u}^{(m)} \cdot \boldsymbol{h}^{(m)} d \Gamma .
$$

In the case of a mixed mortar method there is an additional potential due to the enforced continuity across $\Gamma_{*}$, which is the integral of the unknown surface traction, $\boldsymbol{\lambda}$, dotted with the displacement gap:

$$
\Pi(\boldsymbol{u})^{\operatorname{Lag}}=\int_{\Gamma_{*}} \boldsymbol{\lambda} \cdot[[\boldsymbol{u}]] d \Gamma .
$$

To obtain Nitsche's method, we approximate the surface traction as an average of the stresses on either side of the interface, dotted into the normal. A small penalty traction is added, which would not add energy if the displacement gap were exactly zero everywhere on the surface,

$$
\boldsymbol{\lambda} \approx-\langle\boldsymbol{\sigma}\rangle \cdot \boldsymbol{n}^{(1)}+\frac{\beta}{2}[[\boldsymbol{u}]]
$$

The interfacial potential energy associated with this approach is:

$$
\Pi(\boldsymbol{u})^{N i t}=\int_{\Gamma_{*}}\left(-\langle\boldsymbol{\sigma}\rangle \cdot \boldsymbol{n}^{(1)}+\frac{\beta}{2}[[\boldsymbol{u}]]\right) \cdot[[\boldsymbol{u}]] d \Gamma .
$$


Stationarity of the potential energy imposes that the first variation of the total potential energy, $\Pi(\boldsymbol{u})^{T o t}=\Pi(\boldsymbol{u})+\Pi(\boldsymbol{u})^{N i t}$, be zero. This gives the following variational formulation:

Find $\left(\boldsymbol{u}^{(1)}, \boldsymbol{u}^{(2)}\right) \in \boldsymbol{S}^{(1)} \times \boldsymbol{S}^{(2)}$

$$
\begin{aligned}
& \delta \Pi(\boldsymbol{u})+\delta \Pi(\boldsymbol{u})^{N i t}=0 \\
& \text { for all }\left(\boldsymbol{w}^{(1)}, \boldsymbol{w}^{(2)}\right) \in V^{(1)} \times V^{(2)} .
\end{aligned}
$$

which gives Nitsche's method exactly as it is written in (7). In [15], it is shown that any convex combination of flux operators will yield a consistent method in this context. We take $\langle\boldsymbol{\sigma}\rangle$ to be $\left[\gamma \boldsymbol{\sigma}^{(1)}+(1-\gamma) \boldsymbol{\sigma}^{(2)}\right]$, where $0 \leq \gamma \leq 1$. A generalized form of Nitsche's method for elasticity becomes:

Find $\left(\mathbf{u}^{(1)}, \mathbf{u}^{(2)}\right) \in \boldsymbol{S}^{(1)} \times \boldsymbol{S}^{(2)}$

$$
\begin{gathered}
\sum_{m}^{2} \int_{B^{m}} \boldsymbol{\varepsilon}(\boldsymbol{w})^{(m)}: \boldsymbol{\sigma}^{(m)} d B-\int_{\Gamma_{*}}\left([[\boldsymbol{w}]] \otimes \boldsymbol{n}^{(1)}\right):\left[\gamma \boldsymbol{\sigma}^{(1)}+(1-\gamma) \boldsymbol{\sigma}^{(2)}\right] d \Gamma \\
-\int_{\Gamma_{*}}\left([[\boldsymbol{u}]] \otimes \boldsymbol{n}^{(1)}\right):\left[\gamma \boldsymbol{\sigma}(\boldsymbol{w})^{(1)}+(1-\gamma) \boldsymbol{\sigma}(\boldsymbol{w})^{(2)}\right] d \Gamma+\beta \int_{\Gamma_{*}}[[\boldsymbol{u}]] \cdot[[\boldsymbol{w}]] d \Gamma= \\
\sum_{m=1}^{2} \int_{B^{m}} \boldsymbol{w}^{(m)} \cdot \boldsymbol{f}^{(m)} d B+\sum_{m=1}^{2} \int_{\Gamma_{h}^{(m)}} \boldsymbol{w}^{(m)} \cdot \boldsymbol{h}^{(m)} d \Gamma \\
\text { for all } \boldsymbol{w} \in \boldsymbol{V}^{(1)} \times \boldsymbol{V}^{(2)}
\end{gathered}
$$

A choice of $\gamma=\frac{1}{2}$ returns the original form given by (7).

\section{Discrete Equation Structure}

\subsection{Integration of cut cells}

While the discretization and assembly of the overlapping grid is entirely standard, some special accommodations must be made for the background grid, where some elements may be indiscriminately cut by the surface of the overlapping body. Elements of the underlying grid that are completely covered by the inclusion are considered void and do not contribute to the discrete stiffness equations. In a sharp interface capture technique similar to that used in the extended finite element method [see, for example, 16], elements that are cut by $\Gamma_{*}$ contribute a partial stiffness, due to the uncovered area. All of the degrees of freedom of cut elements remain active, but integration only occurs over the material, or uncovered region of cut elements. It is not possible to use the standard optimal methods of Gauss quadrature (generally two points in each direction for linear quadrilaterals and hexes) over the polygonal, possibly non-convex region that represents the active area. The region is divided into integration triangles (Figure 3), which are evaluated with a sufficiently large Gauss quadrature rule based on the background element interpolations (general linear or bilinear shape functions). 


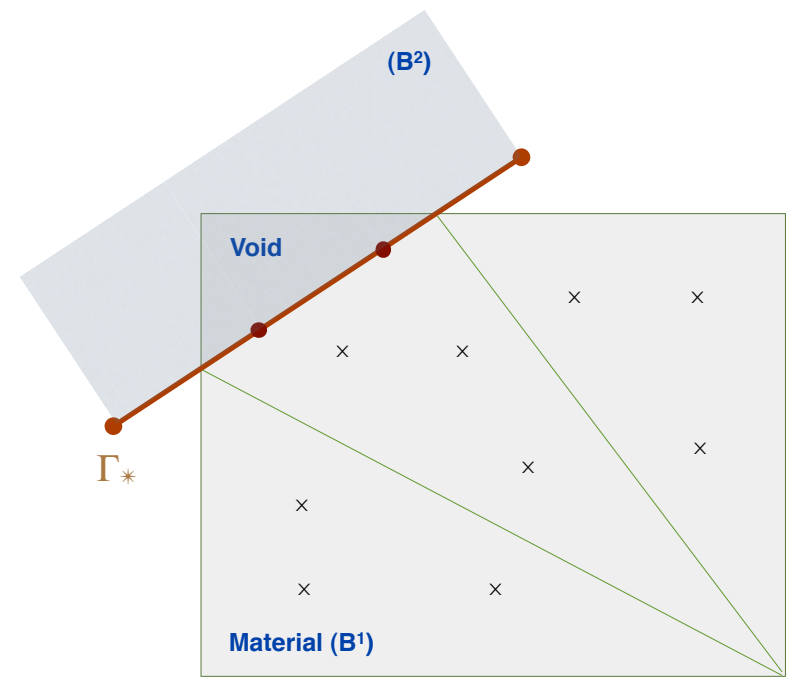

Figure 3: Schematic of partially integrated cut element

The standard expression for a local stiffness matrix for a discrete element integrated with $g$ Gauss points is

$$
\boldsymbol{k}^{e}=\sum_{g=1}^{n_{g}}\left(\boldsymbol{B}^{T}(\boldsymbol{\xi}) \boldsymbol{D} \boldsymbol{B}(\boldsymbol{\xi}) j\right) W_{g}
$$

where $\boldsymbol{\xi}$ are the natural coordinates of a reference element, $j$ is the jacobian of the coordinate transformation from $\boldsymbol{x}$ to $\boldsymbol{\xi}, n_{g}$ is the number of Gauss points, and $W_{g}$ are appropriate Gauss quadrature weights. If we introduce a new coordinate system, $\boldsymbol{r}$, based on the natural coordinates of an integration triangle, the expression for a cut cell divided into $n_{t}$ integration regions is

$$
\boldsymbol{k}_{c u t}^{e}=\sum_{i=1}^{n_{t}}\left(\sum_{l=1}^{n_{g}}\left(\boldsymbol{B}^{T}(\boldsymbol{r}) \boldsymbol{D} \boldsymbol{B}(\boldsymbol{r}) j\right) W_{g}\right)
$$

where $j$ is now the jacobian of the coordinate transformation from $\boldsymbol{x}$ to $\boldsymbol{r}$. Assembly of the modified element stiffness matrices follows standard finite element procedures. There will be some nodes for this technique that lie in the void region of $B^{2}$, but still contribute support to the displacement approximation.

\subsection{Standard mortars}

In this work, we have used either two-dimensional bi-linear quadrilaterals, or linear triangles for the discretization of the bodies. To complete the discretization for the mixed variational statement, we must choose interpolant shape functions for the discrete multipliers $\boldsymbol{\lambda}^{h}$, (as well as their variations, $\boldsymbol{\mu}$ ). The multipliers can be physically interpreted as the traction field over the interfaces enforcing a tied constraint. We have defined this space on the embedded interface, $\Gamma_{*}$, which fixes the definitions of master and slave sides in the mor- 
tar formulation. According to standard terminology, the side on which the multiplier field is defined is the non-mortar, or slave side. The background elements are then the mortar, or master side.

Our choice for an interpolant on the slave surface, $\Gamma_{*}$, is the same field used to discretize the displacement over the surface, that is, piecewise linear shape functions supported by the existing nodes:

$$
\boldsymbol{\lambda}=\sum_{A}^{n^{s}} \bar{N}_{A}^{1}(\boldsymbol{\xi}) \boldsymbol{\lambda}_{A}
$$

where $\boldsymbol{\xi}$ is the piecewise surface parametrization and $n^{s}$ represents the number of surface nodes (i.e., the nodes of body 1 on $\Gamma_{*}$ ). The shape functions $\bar{N}_{A}$ are functions used to interpolate the multipliers over $\Gamma_{*}$; in the baseline mortar method, these are taken to coincide with the surface trace of the standard finite element shape functions $N_{A}$. This choice is simple and convenient, but by no means unique. It is also possible, for example, to take the trace of the master side shape functions, or to judiciously select nodes on the slave surface as supports for the multiplier field, as opposed to using them all.

We will reconsider our expressions for discrete trial functions, and express their restriction to the boundary $\Gamma_{*}$, as

$$
\begin{aligned}
& \boldsymbol{v}^{(1)} \approx \boldsymbol{v}^{(1)^{h}}=\sum_{B} N_{B}^{(1)}\left(\xi^{(1)}\right) \boldsymbol{c}_{B}^{(1)} \\
& \boldsymbol{v}^{(2)} \approx \boldsymbol{v}^{(2)^{h}}=\sum_{C} N_{C}^{(2)}\left(\boldsymbol{\xi}^{(2)}\right) \boldsymbol{c}_{C}^{(2)}
\end{aligned}
$$

As a notational point, $\xi^{(1)}$ are natural parent coordinates of the one-dimensional shape functions formed by the union of slave elements with $\Gamma_{*}$, and $\boldsymbol{\xi}^{(2)}$ are natural parent coordinates of the two-dimensional shape functions from underlying master elements. One may then substitute equations (17), (18), and (19) into the mortar constraint virtual work (4) to obtain the discrete expression:

$$
G^{c}=\sum_{A} \sum_{B} \sum_{C} \boldsymbol{\lambda}_{A} \cdot\left[n_{A B}^{(1)} \boldsymbol{c}_{B}^{(1)}-n_{A C}^{(2)} \boldsymbol{c}_{C}^{(2)}\right]
$$

where the expressions for $n_{A B}^{(1)}$ and $n_{A C}^{(2)}$ are

$$
\begin{aligned}
n_{A B}^{(1)} & =\int_{\Gamma_{*}} N_{A}^{1}\left(\xi^{(1)}\right) N_{B}^{1}\left(\xi^{(1)}\right) d \Gamma \\
n_{A C}^{(1)} & =\int_{\Gamma_{*}} N_{A}^{1}\left(\xi^{(1)}\right) N_{C}^{2}\left(\xi^{(2)}\right) d \Gamma .
\end{aligned}
$$

Remark: Equation (20) contains a coupling between a surface quantity - parametrized by the one-dimensional shape function $N_{A}\left(\xi^{(1)}\right)$, and a volume quantity - parametrized by the two dimensional shape function $N_{C}\left(\boldsymbol{\xi}^{(2)}\right)$. This marks a departure from mortar methods as they 
are generally used for contact mechanics, domain decomposition, or other surface-to-surface constraints.

Evaluation of integrals (21) and (22) are done over mortar subfacets, $\Gamma_{s}$, constructed from segments of the slave surface, $\Gamma_{*}$, containing support from uniquely one master element and one slave element.

\subsubsection{Global equation structure}

The sets $\mathcal{S}^{1}$ and $\mathcal{S}^{2}$ contain the body 1 interface nodes and the body 2 background nodes adjacent to the interface boundary, i.e.,

$$
\mathcal{S}^{1}=\left\{A \mid \boldsymbol{x}_{A}^{1} \in \Gamma\right\}, \quad \mathcal{S}^{2}=\left\{A \mid \boldsymbol{x}_{A}^{2} \in \Omega_{\bar{e}}\right\}, \text { and } \bar{e}=\left\{e \mid B_{e}^{2} \cap \Gamma\right\} .
$$

In addition, let the vector $\overline{\boldsymbol{d}}^{1}$ be the collection of nodal displacements $\overline{\boldsymbol{d}}_{A}^{1}, A \in \mathcal{S}^{1}$ and the vector $\overline{\boldsymbol{d}}^{2}$ of nodal displacements $\overline{\boldsymbol{d}}_{A}^{2}, A \in \mathcal{S}^{2}$. Using these definitions, and assuming that $\bar{N}_{A}$ are the normal element shape functions $N_{A}$, substitution of (17) in (3) yields the discrete constraint matrix equations corresponding to the standard mortar method

$$
G^{1} \bar{d}^{1}-G^{2} \bar{d}^{2}=0
$$

where matrices $\boldsymbol{G}^{1}$ and $\boldsymbol{G}^{2}$ are defined as ${ }^{3}$

$$
\boldsymbol{G}^{1}=\left[G_{A B}^{1}\right]=\sum_{s=1}^{n_{s}}\left(n_{A B}^{(1)}\right)_{\Gamma_{s}}, \quad G^{2}=\left[G_{A C}^{1}\right]=\sum_{s=1}^{n_{s}}\left(n_{A C}^{(2)}\right)_{\Gamma_{s}} \quad \forall A, B \in \mathcal{S}^{1}, C \in \mathcal{S}^{2}
$$

To illustrate the imposition of the interface constraints in terms of global equation structure, we partition the nodes in terms of solid interface nodes $\mathcal{S}^{1}\left(23_{1}\right)$, body 2 solid nodes adjacent to interface $\mathcal{S}^{2}\left(23_{2}\right)$ and all other nodes $A \backslash\left(\mathcal{S}^{1} \cup \mathcal{S}^{2}\right)$. This partitioning yields the following discrete matrix equations

$$
\left[\begin{array}{cccc}
\boldsymbol{K}^{*} & \boldsymbol{K}^{*, 1} & \boldsymbol{K}^{*, 2} & 0 \\
{\left[\boldsymbol{K}^{*, 1}\right]^{T}} & \overline{\boldsymbol{K}}^{1} & 0 & {\left[\boldsymbol{G}^{1}\right]^{T}} \\
{\left[\boldsymbol{K}^{*, 2}\right]^{T}} & 0 & \overline{\boldsymbol{K}}^{2} & -\left[\boldsymbol{G}^{2}\right]^{T} \\
0 & \boldsymbol{G}^{1} & -\boldsymbol{G}^{2} & 0
\end{array}\right]\left\{\begin{array}{l}
\boldsymbol{d} \\
\overline{\boldsymbol{d}}^{1} \\
\overline{\boldsymbol{d}}^{2} \\
\boldsymbol{\lambda}
\end{array}\right\}=\left\{\begin{array}{l}
\boldsymbol{f}^{(e x t)} \\
\overline{\boldsymbol{f}}^{(e x t)^{1}} \\
\overline{\boldsymbol{f}}^{(e x t)^{2}} \\
\mathbf{0}
\end{array}\right\}
$$

where $\boldsymbol{K}^{*}$ is the stiffness associated with nodes $A \backslash\left(\mathcal{S}^{1} \cup \mathcal{S}^{2}\right)$ and $\boldsymbol{K}^{*, i}$ is the stiffness coupling nodes in $\mathcal{S}^{i}$ to the forces associated with non-interface nodes, for $i=1,2$. The partitioning is such that body 1 interface nodes occupy the second row in (26), body 2 interface nodes occupy the third row in (26) and all other nodes occupy the first row. The fourth row incorporates the constraint equations. The quantities $\overline{\boldsymbol{f}}^{(e x t)^{1}}$ and $\overline{\boldsymbol{f}}^{(e x t)^{2}}$ in (26) represent the externally

\footnotetext{
${ }^{3}$ Actually, we abuse notation slightly here, as the elements of the $\boldsymbol{G}^{i}$ are given in terms of $G_{A B}^{i} \boldsymbol{I}$, where $\boldsymbol{I}$ is the $2 \times 2$ identity matrix in two dimensions, and $3 \times 3$ in three dimensions. We will continue this slight notational abuse throughout the article.
} 
imposed forces involving interface nodes. The computation of quantities associated with $\mathcal{S}^{2}$ must reflect the partially filled elements (see Figure 3) due to the overlapping mesh of body 1 .

\subsection{Penalty method}

The discrete form of the penalty method involves evaluating the expression for virtual work associated with the penalty method,

$$
G^{P}=\beta \int_{\Gamma_{*}}[[\boldsymbol{u}]] \cdot[[\boldsymbol{w}]] d \Gamma .
$$

To expand the expression, we need to be explicit about the restriction of the displacements to $\Gamma_{*}$ :

$$
\begin{gathered}
\boldsymbol{u}^{(1)} \approx \boldsymbol{u}^{(1)^{h}}=\sum_{D} N_{D}^{(1)}\left(\xi^{(1)}\right) \overline{\boldsymbol{d}}_{D}^{(1)} \\
\boldsymbol{u}^{(2)} \approx \boldsymbol{u}^{(2)^{h}}=\sum_{E} N_{E}^{(2)}\left(\boldsymbol{\xi}^{(2)}\right) \overline{\boldsymbol{d}}_{E}^{(2)}
\end{gathered}
$$

The global system of equations becomes

$$
\left[\begin{array}{ccc}
\boldsymbol{K}^{*} & \boldsymbol{K}^{*, 1} & \boldsymbol{K}^{*, 2} \\
{\left[\boldsymbol{K}^{*, 1}\right]^{T}} & \overline{\boldsymbol{K}}^{1}+\boldsymbol{K}_{p}^{(1,1)} & \boldsymbol{K}_{p}^{(1,2)} \\
{\left[\boldsymbol{K}^{*, 2}\right]^{T}} & \boldsymbol{K}_{p}^{(2,1)} & \overline{\boldsymbol{K}}^{2}+\boldsymbol{K}_{p}^{(2,2)}
\end{array}\right]\left\{\begin{array}{l}
\boldsymbol{d} \\
\overline{\boldsymbol{d}}^{1} \\
\overline{\boldsymbol{d}}^{2}
\end{array}\right\}=\left\{\begin{array}{l}
\boldsymbol{f}^{(e x t)} \\
\overline{\boldsymbol{f}}^{(e x t)^{1}} \\
\overline{\boldsymbol{f}}^{(e x t)^{2}}
\end{array}\right\}
$$

where the global quantities are assembled as, for example,

$$
\boldsymbol{K}^{(1,1)}=\left[K_{B D}^{(1,1)}\right]=\int_{\Gamma_{*}} N_{B}^{(1)} N_{D}^{(1)} d \Gamma_{*},
$$

and similarly for the other terms.

\subsection{Nitsche's method}

To form the global stiffness for Nitsche's method we must evaluate the extra virtual work terms in equation (14) in a discrete setting:

$$
\begin{aligned}
G^{N}= & \boldsymbol{K}^{N} \boldsymbol{d}+\left(\boldsymbol{K}^{N}\right)^{T} \boldsymbol{d} \\
= & -\int_{\Gamma_{*}}[[\boldsymbol{w}]] \otimes \boldsymbol{n}^{(1)}:\left[\gamma \boldsymbol{\sigma}^{(1)}+(1-\gamma) \boldsymbol{\sigma}^{(2)}\right] d \Gamma \\
& -\int_{\Gamma_{*}}[[\boldsymbol{u}]] \otimes \boldsymbol{n}^{(1)}:\left[\gamma \boldsymbol{\sigma}(\boldsymbol{w})^{(1)}+(1-\gamma) \boldsymbol{\sigma}(\boldsymbol{w})^{(2)}\right] d \Gamma .
\end{aligned}
$$

We describe the local calculations necessary to evaluate the first integral and take advantage of the fact that second is the transpose of the first, due to the matching spaces for 


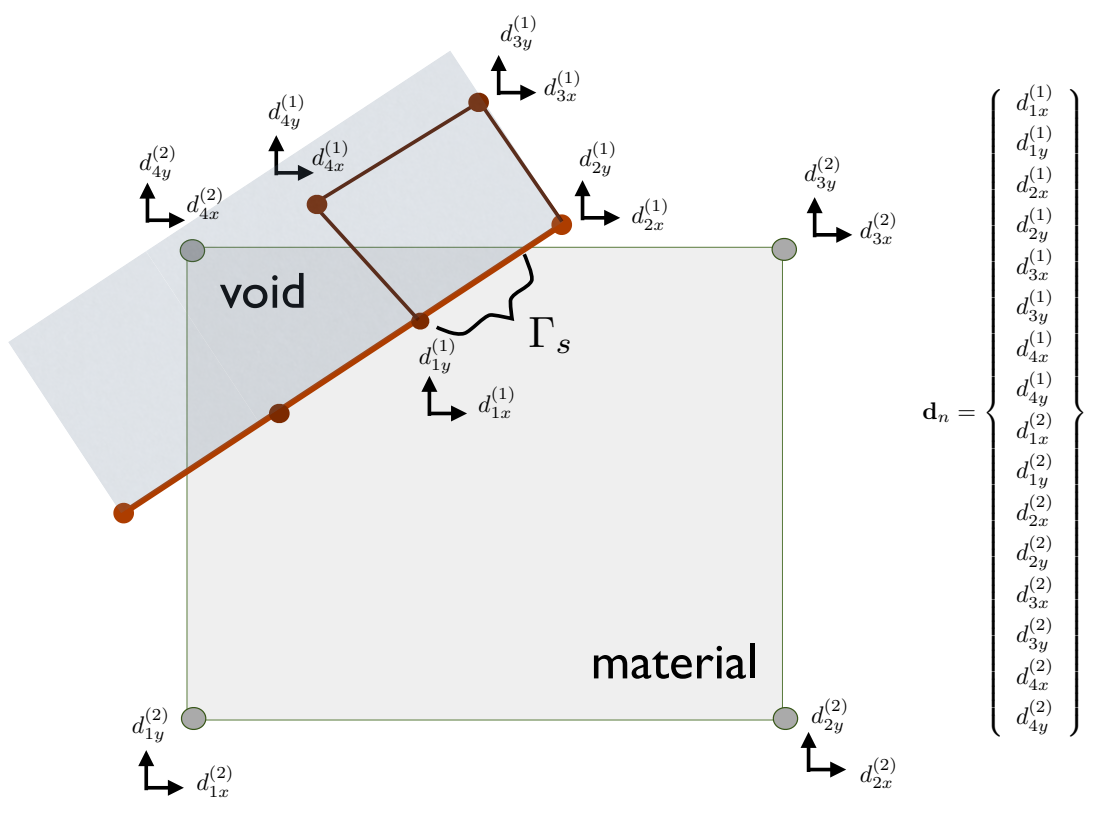

Figure 4: Degrees of freedom which contribute to Nitsche interfacial stiffness as calculated over one mortar segment

displacements and their variations. As in the case of the standard mortars and the penalty method, it will make sense to evaluate the discrete integral over individual mortar sections. In this case, however, there are extra degrees of freedom pulled into the calculation over a single mortar, due to presence of the stress operators on both sides of the interface. For convenience, we introduce a special vector of local nodal displacements, $\boldsymbol{d}_{n, s}$ associated with a single mortar segment, $s$, corresponding to all of the degrees of freedom involved both in the discrete gap function, and the slave and master side stress operators for elements associated with that segment (1 each, master and slave). Figure 4 shows an example cut element and resulting modified local displacement vector.

The local discrete stiffness that results from plugging our discrete approximations for both $\boldsymbol{u}^{(m)}$ and $\boldsymbol{\sigma}^{(m)}$ for a single mortar segment, $s$, into the first integral of (32) is

$$
\mathbf{k}^{N, s}=\left[k_{p q}^{s}\right]=\int_{\Gamma_{s}}\left(N_{A}^{(1)}-N_{E}^{(2)}\right)\left[\gamma C_{i j k l}^{(1)} N_{B, l}^{(1)}+(1-\gamma) C_{i j k l}^{(2)} N_{F, l}^{(2)}\right] n_{j}^{(1)} d \Gamma .
$$

Indexing is done via

$$
p=2(A \text { or } E-1)+i \text { and } q=2(B \text { or } F-1)+k,
$$

where $A$ indexes over the nodes which support the gap function on $\Gamma_{*}$, and $B$ and $F$ index over all of the local degrees of freedom in the vector $\boldsymbol{d}_{n, s}$ associated with the slave and master sides respectively. $N_{A}^{(1)}$ is restricted to those shape functions associated with the slave side, 
and $N_{E}^{(2)}$ is similarly restricted to master side nodes.

\subsubsection{Global equation structure}

The coupling of additional internal degrees of freedom in the local operator means that the global equation structure for Nitsche's method unfortunately does not fit conveniently in

the partitioning scheme introduced for the mortar and penalty formulations. We can write the global system in the following way:

$$
\boldsymbol{K}+\boldsymbol{K}^{N}+\left(\boldsymbol{K}^{N}\right)^{T}+\boldsymbol{K}^{S}=\boldsymbol{F}
$$

where

$$
\boldsymbol{K}^{n}=\underset{s=1}{n_{s}}\left(\boldsymbol{k}^{N, s}\right)
$$

and the "stabilization" term, $\boldsymbol{K}^{S}$, is evaluated in exactly the same manner as the traditional penalty term. ${ }^{4}$

The choice of the parameter $\beta$ is important to the stability of the problem. We refer to $\beta$ as a stabilization parameter in this case rather than a penalty parameter because it no longer acts primarily to enforce the constraint. Indeed, Nitsche's method with any value of $\gamma$ is able to pass a patch test exactly for any value of $\beta$, including zero. However, in the discrete sense, the subtraction of the extra interface terms leads to a global set of equations that is no longer guaranteed to be positive definite. This is related to the loss of coercivity in the functional form of the method. The penalty style operator restores coercivity (in the continuous problem), or positive definiteness (in the discrete problem) to the system of equations. A stability parameter that is too small produces unstable results, whereas a stability parameter that is too large essentially forces the problem to behave as a penalty method, which produces the same locking problems as typical Lagrange multipliers. For our numerical examples, it was easy to determine an appropriate value based on the coarsest meshes, starting with an estimate based on the stiffness of background elements, and increasing slightly if the discrete system failed a positive definiteness test. The value of the stability parameter scales with mesh refinement by $\frac{1}{h}$ where $h$ is the local mesh size. For more general cases, efficient methods to estimate optimal parameters are presented in [8] and [11].

\section{Numerical Examples}

Though ultimately intended for threeidimensional use, algorithms based on the preceding methods were prototyped into a two-dimensional MATLAB code with linear triangle and quadrilateral finite elements. Finite element meshes were created with CUBIT, from Sandia National Laboratories (cubit.sandia.gov).

\footnotetext{
${ }^{4}$ The forms of penalty stabilization are not unique, results here use the standard mortar integrals from equations (21) and (22). Results using alternate forms, such as constant pressures, showed little influence on results.
} 


\subsection{Pure bending}

A simple example can demonstrate the strengths and weakness of all of the proposed methods. As shown in Figure 5(a), we construct a rectangular $[1 \times 1.5]$ background domain (domain 2), with the origin at the center of the left boundary, and embed a smaller $[1 \times$ 0.625] rectangular body (domain 1), in left portion, so that the boundaries coincide on three surfaces, and continuity only needs to be enforced on a vertical surface at $x=0.625$. The crosshatch mesh in Figure 5(a) is constructed to represent a "worst case scenario" for the locking phenomenon, with the constrained surface cutting diagonally across the background mesh. Figure 5(a) shows a non-uniform background mesh. Both uniform and non-uniform meshes were used. Stress contours will generally be shown on uniform meshes, while the convergence studies are done with non-uniform grids for generality.

A linear distribution of normal forces on the left and right faces should result in bending stresses dependent only on $y$ (Figure 5(b)), regardless of the material parameters of the problem. In this case, the material properties are either uniform throughout the entire domain $\left(E_{1}=E_{2}=50, \nu_{1}=\nu_{2}=0.3\right)$, or comparatively stiff in the overlapping section $\left(E_{1}=50000, E_{2}=50\right)$. The equivalent moment exerted by the external loads is $M=1.5$. Units are deliberately left out here, given that they can be consistently chosen in any system.

The following cases are considered for the mortar, penalty and Nitsche results in this section:

1. A fine mesh overlaying a coarser mesh, with the same material parameters for both bodies.

2. Overlapping and underlying meshes that are comparable in terms of mesh density, with an embedded stiffness 1000 times greater than the underlying stiffness.

3. A fine mesh overlaying a coarser mesh with an embedded stiffness 1000 times greater than the underlying stiffness

Examination of Figure 6 shows that the mortar method works very well for cases (1) and (2) but shows highly oscillatory stresses due to mesh locking at the interface for case (3).

Figure 7 studies the behavior of the penalty method for case (3). For low penalty numbers, the stress field appears qualitatively improved over the mortar method, though as the penalty number increases for the same mesh, locking reappears.

Finally, the weighted $\left(\gamma=\frac{E_{2}}{E_{1}+E_{2}}\right)$ and single-sided $(\gamma=0)$ Nitsche methods demonstrate good results for case (3) in Figure 8 and 9 respectively

The correct choice of a stabilization parameter is important for accuracy. A stabilization parameter which is too low will not guarantee positive definiteness of the global system of equations, which is important for a stable result. A parameter which is too high, however, will drive the penalty style term in Nitsche's method to dominate the constraint enforcement. At high penalty parameters, the penalty method starts to behave similar to the mortar method, so high values of penalty $\alpha$ and stabilization $\beta$ can produce the same locking effect seen in the standard mortar case. Plots of the value of the stabilization parameter versus the error in terms of both L2 and energy norms are given in Figures 10 for the weighted Nitsche's method 


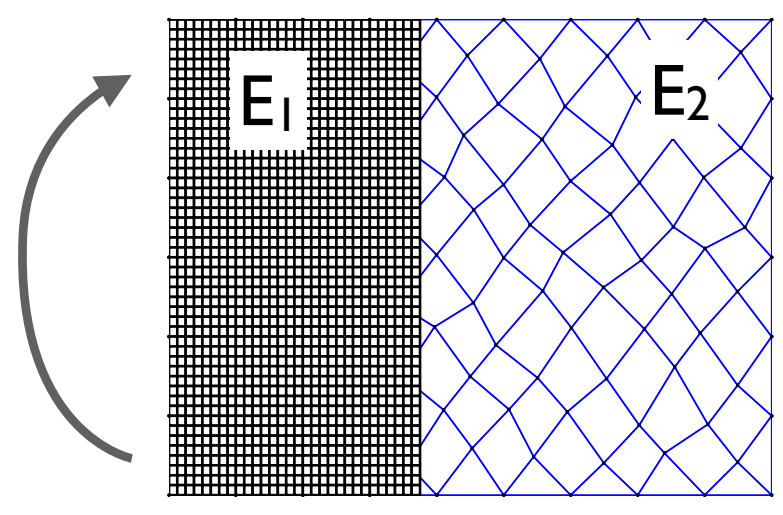

(a)

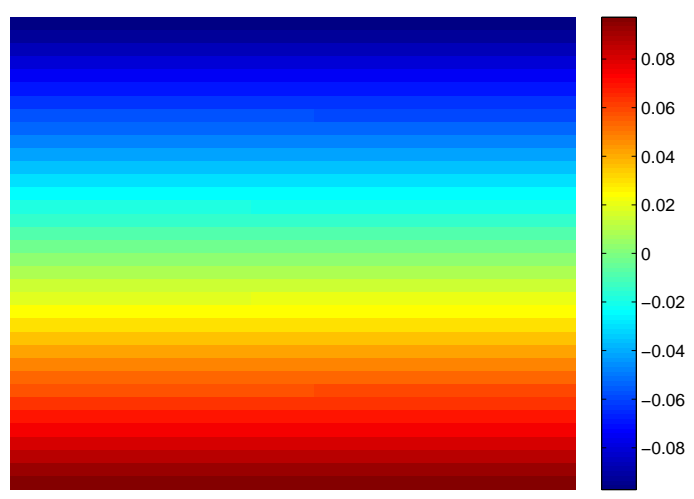

(b)

Figure 5: (a) Problem setup for an embedded mesh in beam bending, (b) analytical solution. Both uniform and non-uniform (as shown) meshes were used

for case (3), and demonstrate the idea that there exists a best value for the parameter. The worst error is seen at values just below the positive definiteness threshold, and the greatest accuracy tends to occur with $\beta$ only slightly greater. For case (3), a value of $\beta=2000$ gives good accuracy for Nitsche's method.

For this particular study, a value of $\gamma=0$ gave very similar results to the weighted method (Figure 9), although this is not always the case. It is worth noting that the standard application of Nitsche's method using a perfect average $(\gamma=0.5)$ is problematic. In this case, the penalty number has to be very high to overcome the coercivity positive-definiteness threshold. The non-physical oscillations in stress in Figure 11(a), come from a highly nonpositive definite discrete system ${ }^{5}$. When the number is high enough, however, to overcome the restriction, the locking behavior is already present (Figure 11(b)). In short, for case (3) and $\gamma=0.5$ no range of $\beta$ produces good results.

Convergence plots for L2 displacement and energy norms are given in Figure 12 for the problematic case (3) for both the mortar, penalty, and Nitsche's formulation. For generality, convergence plots are based on data from unstructured background grids. Since the ordinary metrics for convergence can be deceiving in the consideration of a two-body problem in which the stiffness of one body is much greater than the stiffness of the other, we use a scaled form of the standard error norms for the bulk displacement norm

$$
\|\boldsymbol{e}(\boldsymbol{x})\|_{L_{2}}=\left(E_{2} \int_{B^{1}} \boldsymbol{e} \cdot \boldsymbol{e} d B+E_{1} \int_{B^{2}} \boldsymbol{e} \cdot \boldsymbol{e} d B\right)^{1 / 2},
$$

\footnotetext{
${ }^{5}$ The method is highly non-coercive due to the terms in the last line of Eq. 32. There, the gap [[u]] is being integrated against the stiff foreground term $\gamma \sigma(w)^{(1)}$ term yielding acute "negative" work.
} 
where $\boldsymbol{e}=\boldsymbol{u}^{h}-\boldsymbol{u}$, and an energy norm,

$$
\|\boldsymbol{e}(\boldsymbol{x})\|_{e}=\left(\int_{\Omega}\left(\boldsymbol{\sigma}^{h}-\boldsymbol{\sigma}\right):\left(\boldsymbol{\sigma}^{h}-\boldsymbol{\sigma}\right) d \Omega\right)^{1 / 2} .
$$

The standard mortar method shows optimal convergence in the L2 displacement norms (though with poor accuracy). They do not, however, converge in the energy norm. The penalty method appears to function very well in terms of convergence in the energy norms (see Figure 12), which is not surprising given the excellent stress fields. The method does not, however, converge optimally in the L2 norm of displacement. In both cases it is necessary to scale the parameter with the mesh size, $h$, at a rate of $\alpha \propto \frac{1}{h}$. The weighted Nitsche's methods with both choices of the parameter $\gamma$ give the correct rates in both norms, with little extra cost.

\subsection{Example in curved interfaces}

Numerical interface methods are typically easier for straight surfaces over curved ones. A good example is the general inability of the (numerical) mortar implementation to exactly pass patch tests on curved interfaces. For this reason two more examples were constructed to test the methods for a composite bodies with curved interfaces. The first is shown in Figure 13(a). It is, roughly speaking, a stiff thick walled cylinder ( $B^{1}$ with $E_{1}=50000$, $\nu=0.3)$, and a soft, nearly incompressible material inside $\left(B^{1}\right.$ with $\left.E_{1}=50, \nu=0.45\right)$. Locking is most apparent using the mortar method in the case that there is a variable stress field along the interface. Thus, the boundary conditions involve a changing pressure field on the exterior of the cylinder.

With no analytical solution at hand for this case, comparisons are made to a refined finite element mesh in which the interface between the two materials is explicitly gridded. Figure 13 shows radial stress contours for the standard solution, a mortar interface method in which locking is apparent at the embedded interface, and a weighted Nitsche method solution. Stress contours for a penalty method and a single-sided Nitsche method are qualitatively indistinguishable from the weighted Nitsche's method.

\subsection{Multiple inclusions}

In the final example, three inclusions, one straight and two curved, are embedded in a background grid. Two of the inclusions are stiffer than the surrounding material, and one is significantly softer. Material properties and boundary conditions are shown in Figure 14. The domain is loaded in the vertical direction via a non-uniform exterior load. A reference solution for the given boundary conditions is shown via a refined conforming mesh (Figure 15). The solution in terms of normal yy stresses is given in Figure 16 for standard mortars, Figure 17 for the penalty method, and Figure 18 for a weighted Nitsche's method. A single-sided method is not studied for this case because for one inclusion the background grid functions as the "stiff" side whereas in the other two it is the "soft" side. Though it is obviously possible for a different "side" to be chosen for different inclusions, it is easier

simply to allow the weighting parameter, $\gamma$ to automatically address the issue of which side 


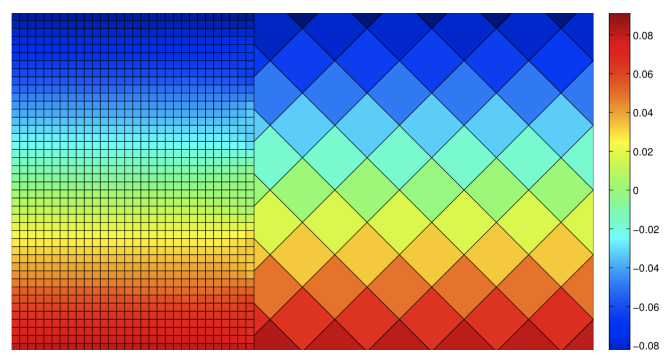

(a) Case (1) bending stresses

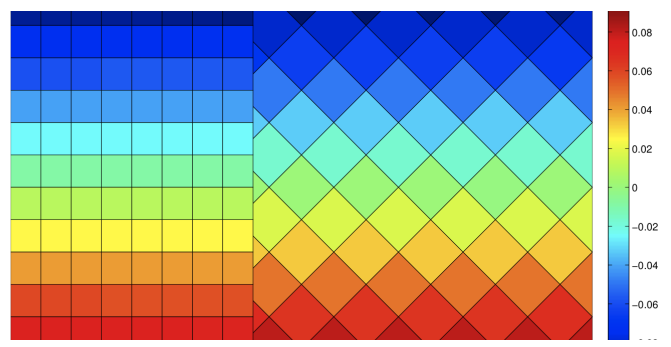

(c) Case (2) bending stresses

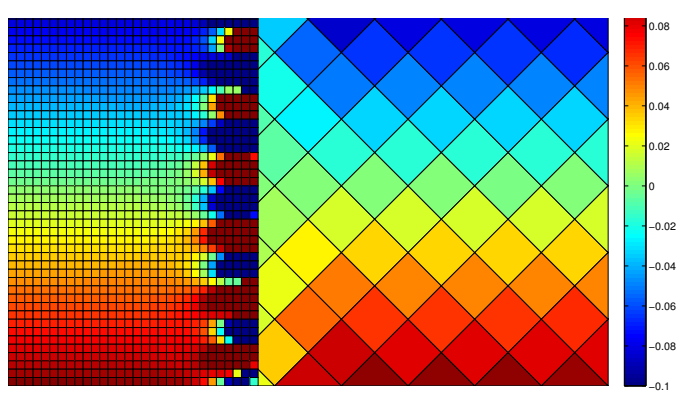

(e) Case (3) bending stresses

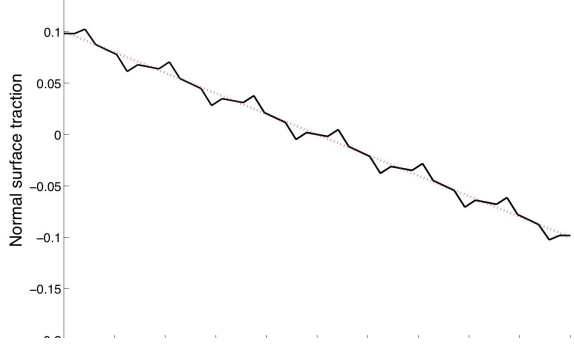

(b) Case (1) surface tractions

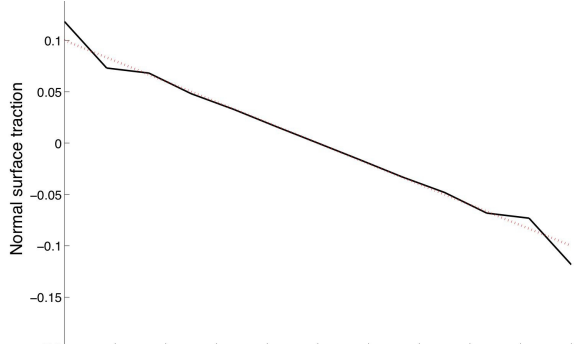

(d) Case (2) surface tractions

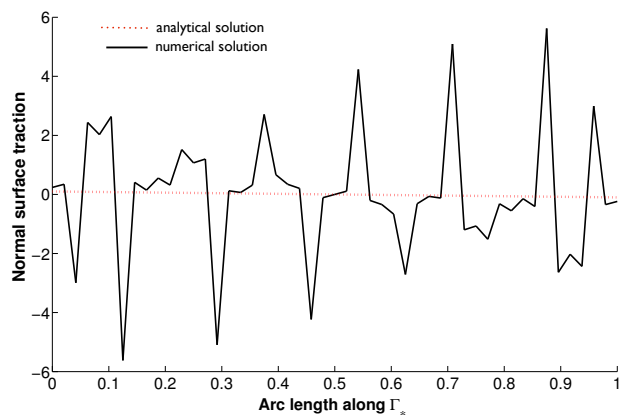

(f) Case (3) surface tractions

Figure 6: Standard mortar constraints

of the interface is stiffer, and bias the method to the softer material. Convergence plots are shown for the multiple inclusion problem in Figure 19.

As in the first example, mortar methods do not attain optimal convergence in the L2 norm, while penalty methods do not achieve an optimal rate in the energy norm. The weighted Nitsche's method is the only method that exhibits fully optimal convergence rates for both norms. A final interesting results is seen from setting the inclusions to the same material properties as the background, and applying a uniform load, essentially performing a curved surface patch test. For curved inclusions, Nitsche's method is the only of the methods that returns the reference solution to machine precision. 


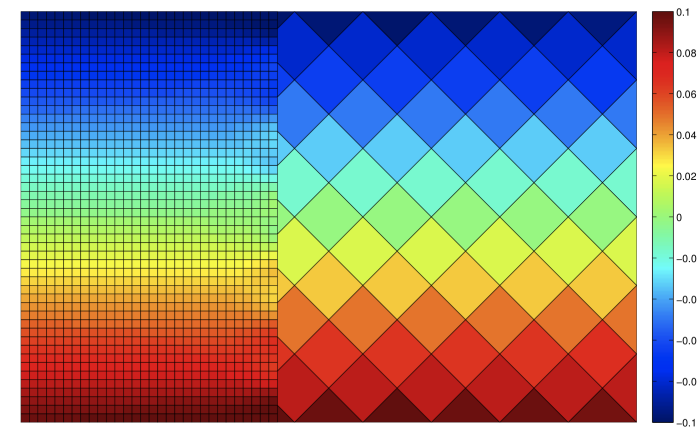

(a) Case (3) bending stresses, $\alpha=2,000$

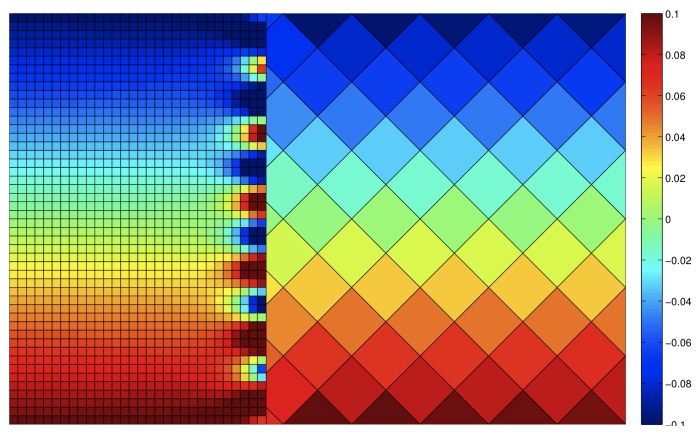

(c) Case (3) bending stresses, $\alpha=50,000$

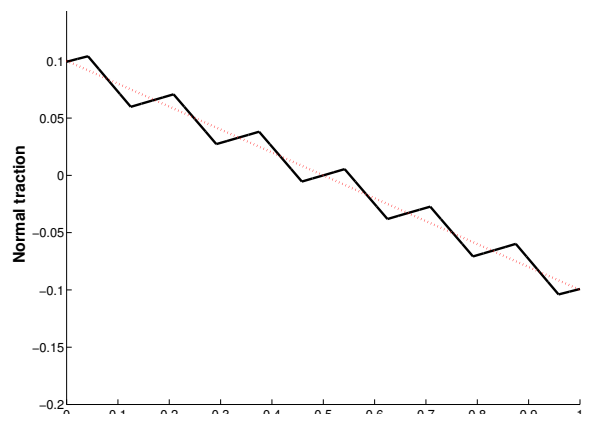

(b) Case (3) surface tractions, $\alpha=2,000$

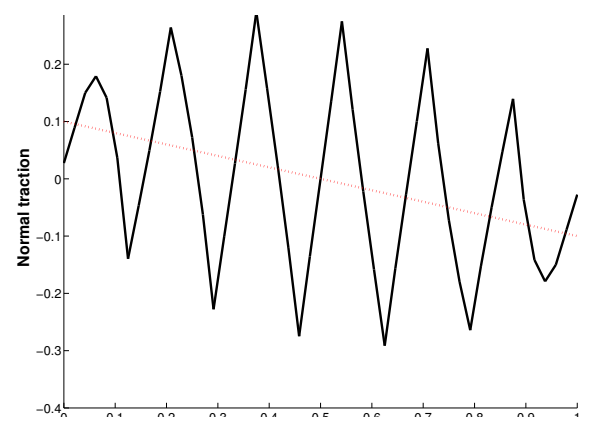

(d) Case (3) surface tractions, $\alpha=50,000$

Figure 7: Penalty method

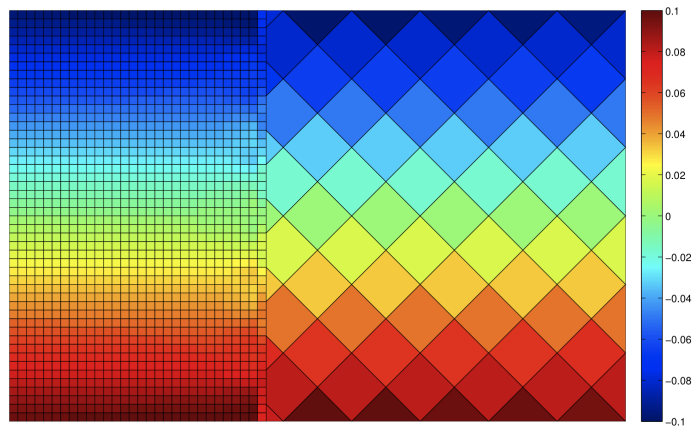

(a) Case (3) bending stresses, $\beta=2,000$

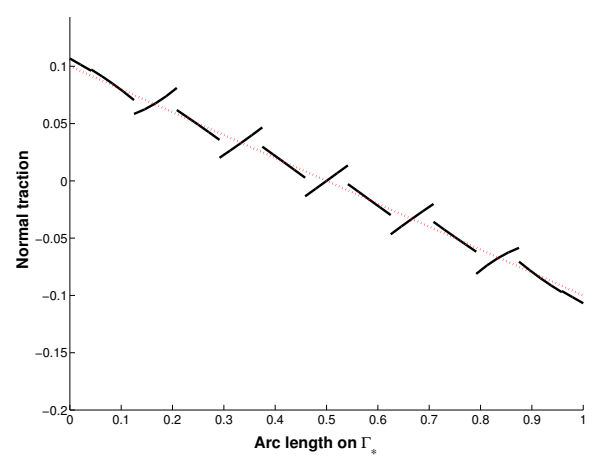

(b) Case (3) surface tractions, $\beta=2,000$

Figure 8: Weighted Nitsche's method $\left(\gamma=\frac{E_{2}}{E_{1}+E_{2}}\right)$ 


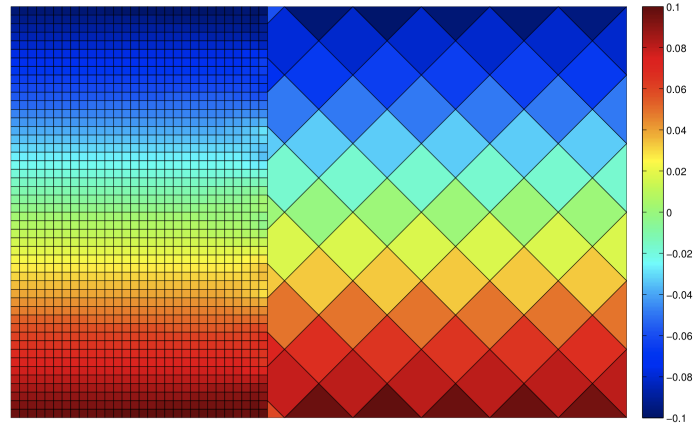

(a) Case (3) bending stresses, $\beta=1,750$

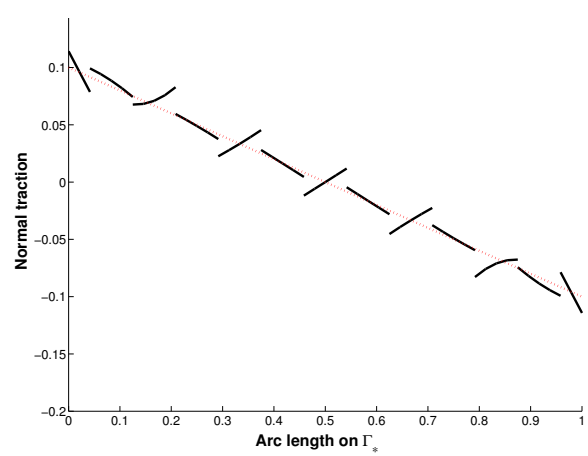

(b) Case (3) surface tractions, $\beta=1,750$

Figure 9: Single-sided Nitsche's method $(\gamma=0)$ 


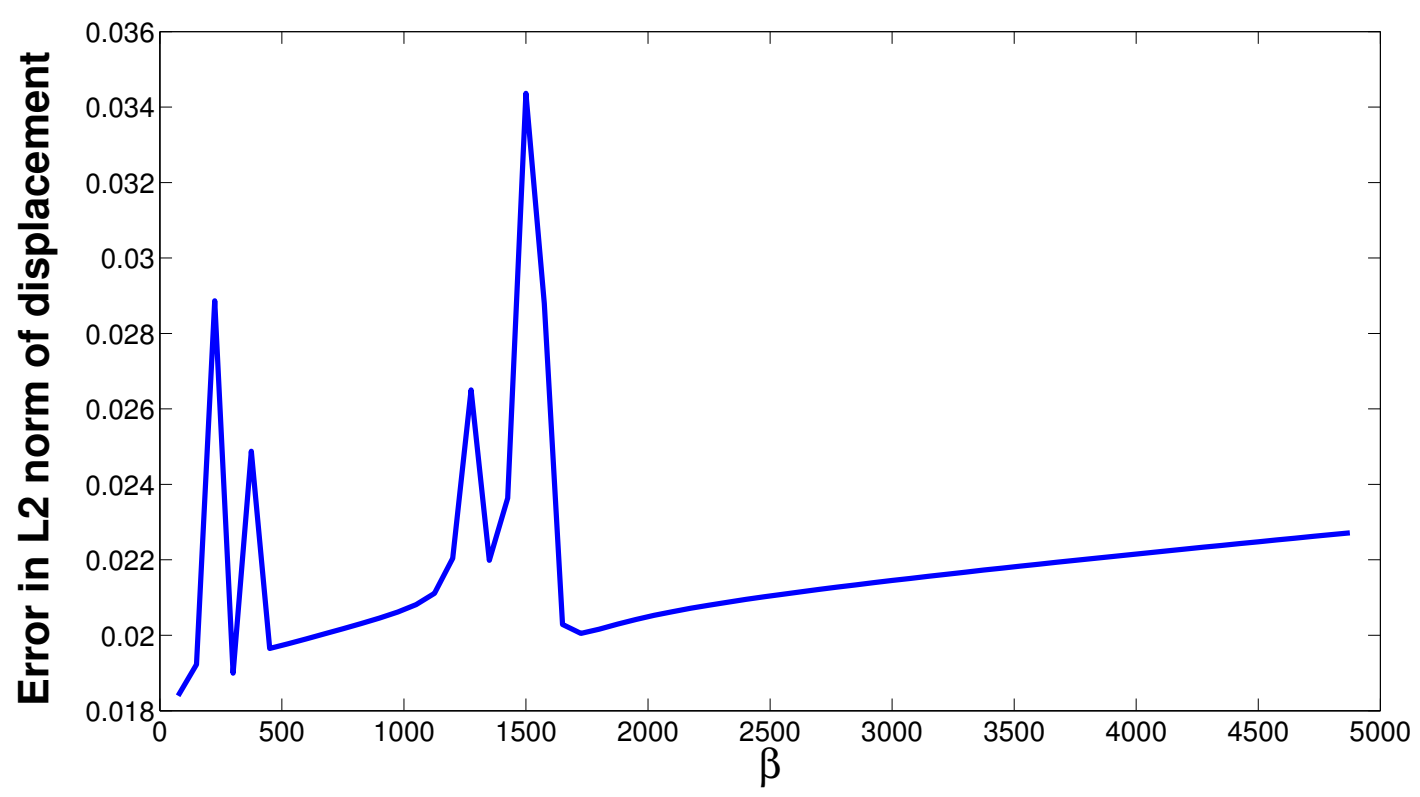

(a)

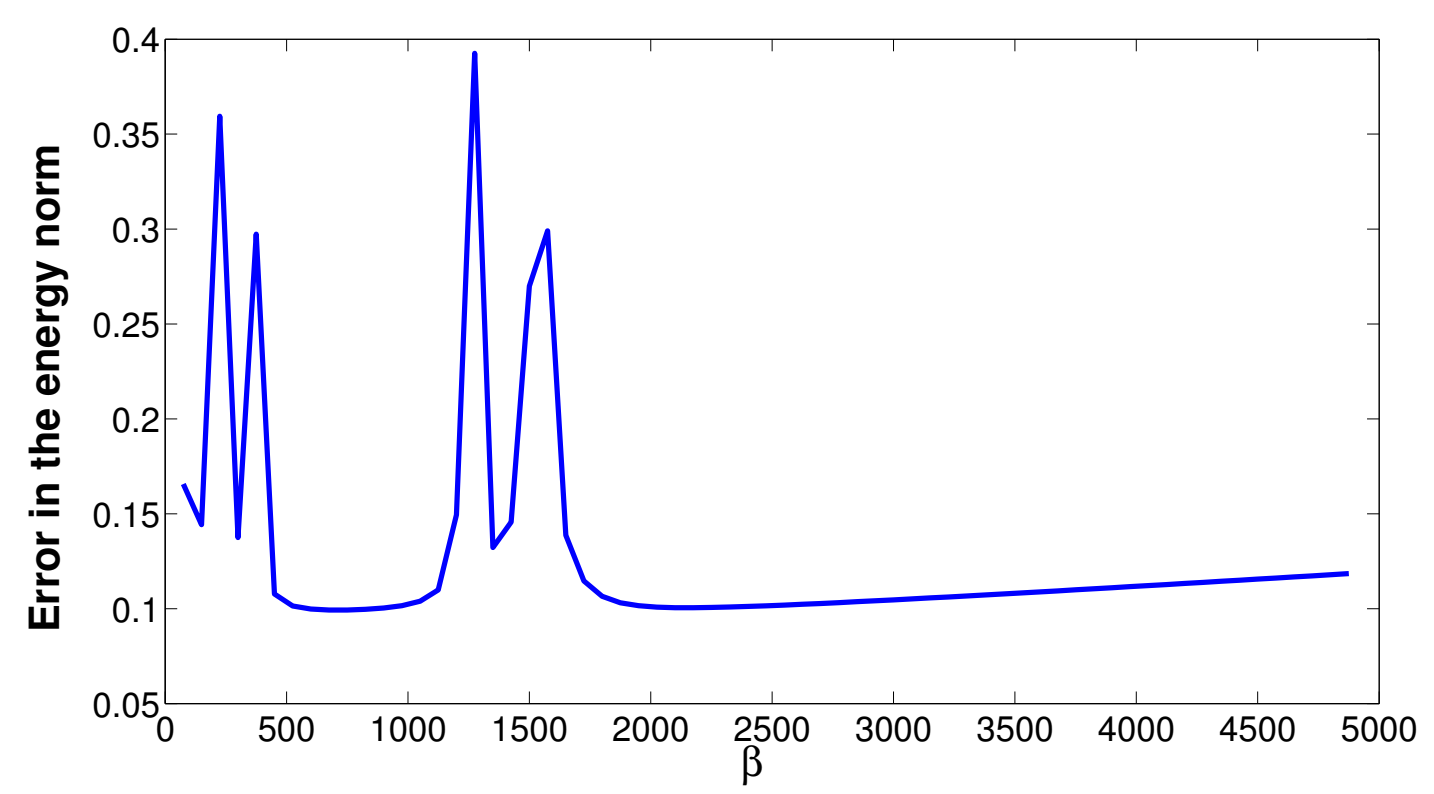

(b)

Figure 10: Error norms versus stabilization parameter for the weighted Nitsche's method, case (3) 


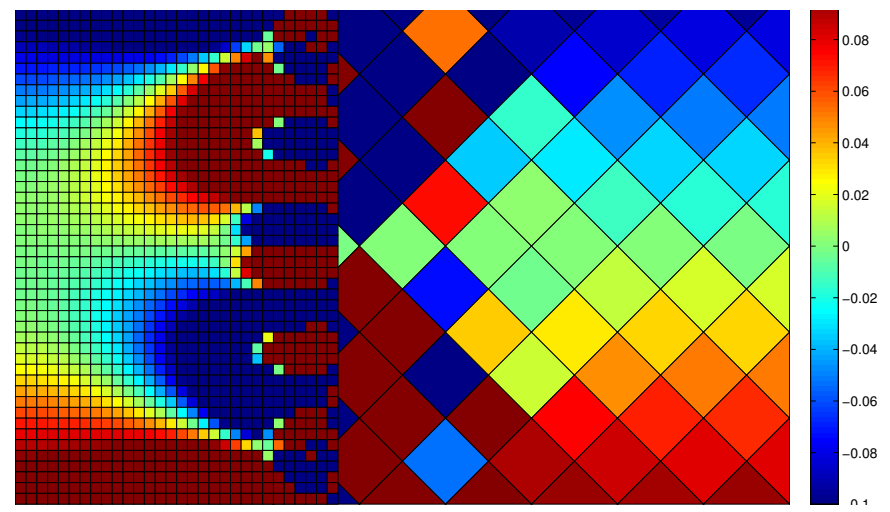

(a) Case (3) bending stresses, $\beta=10,000$

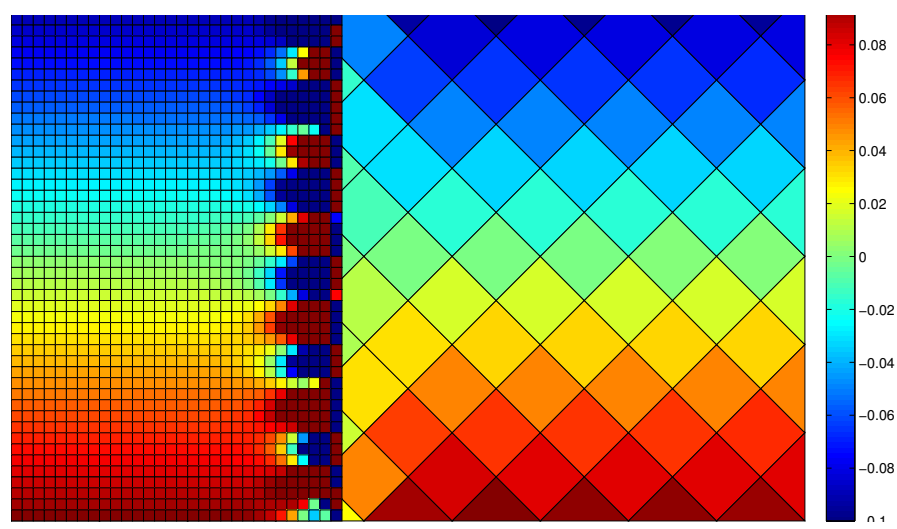

(b) Case (3) bending stresses, $\beta=1,000,000$

Figure 11: Classic $(\gamma=0.5)$ Nitsche's method 


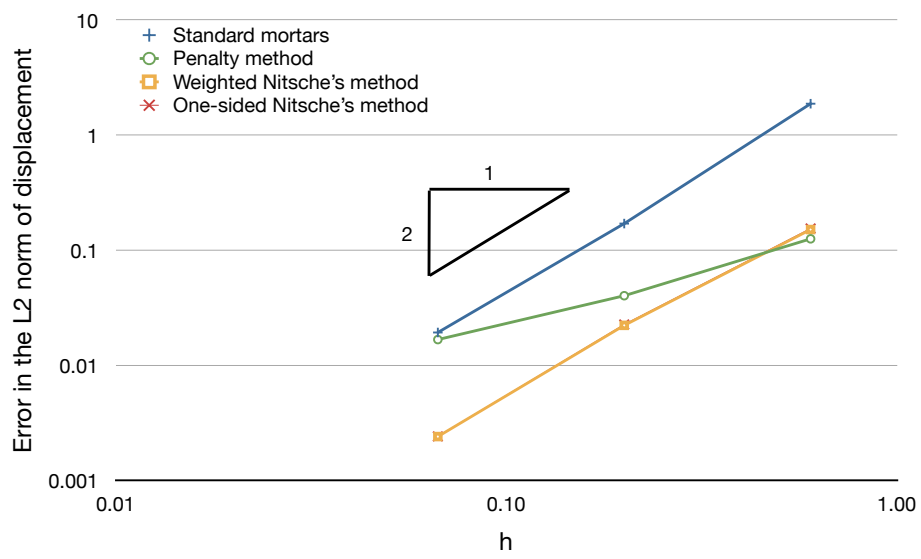

(a)

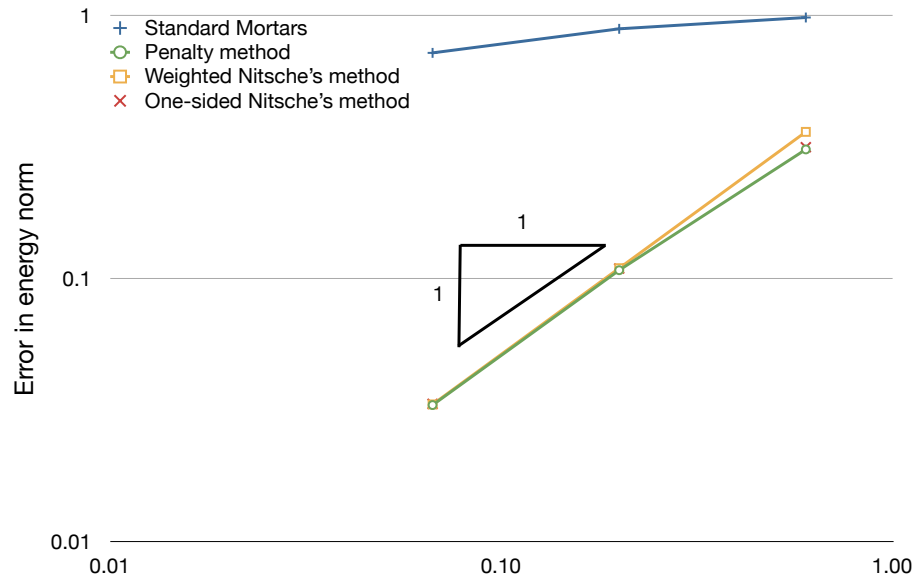

(b)

Figure 12: Convergence rates of the various methods; for linear elements, optimal slope in the L2 norm is 2 (a). Optimal slope in the energy norm is 1 (b). 


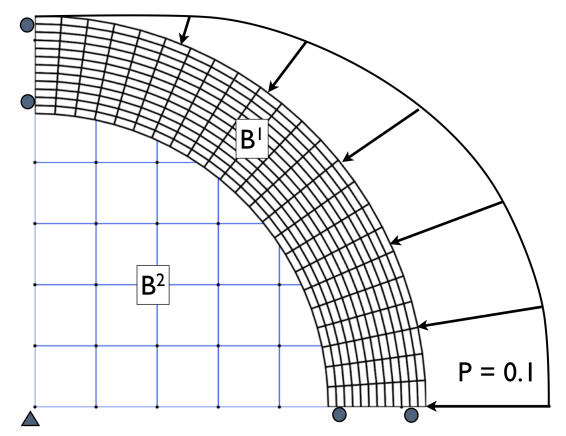

(a) Problem setup

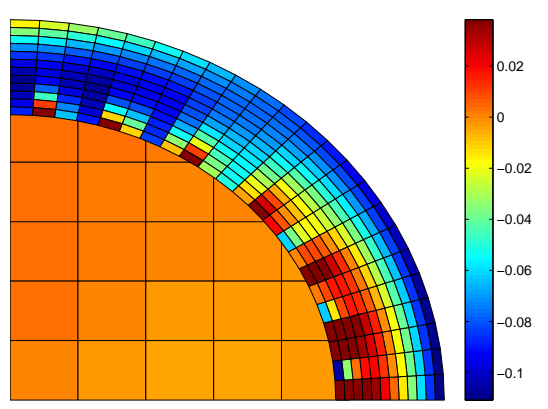

(c) Standard mortars

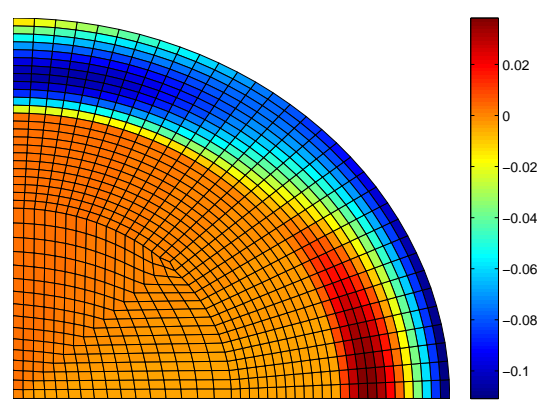

(b) Conforming mesh

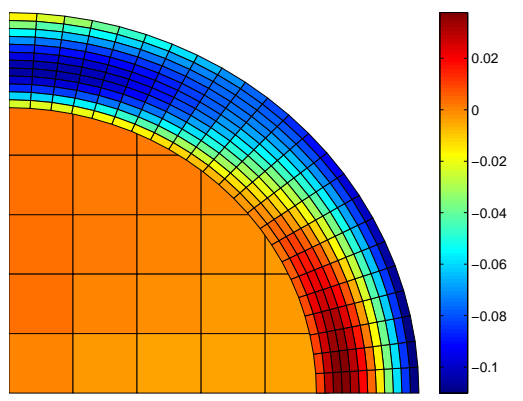

(d) Nitsche's method

Figure 13: Curved interface example

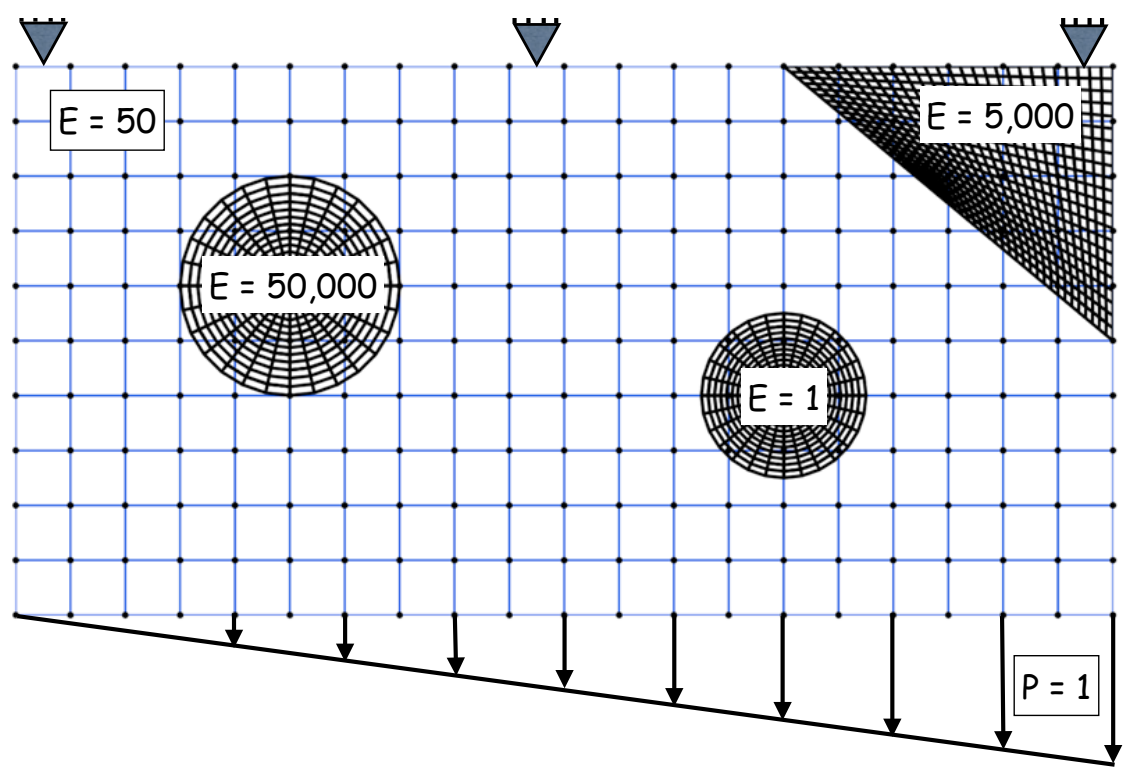

Figure 14: Problem setup, multiple inclusion example 


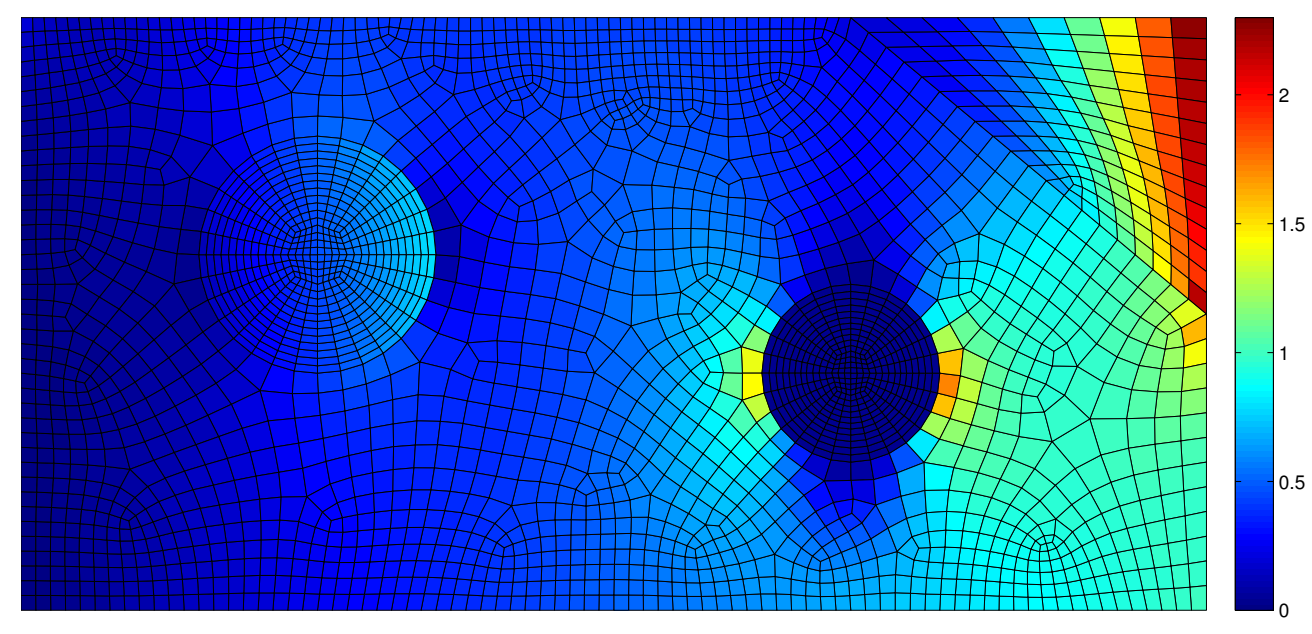

Figure 15: Conforming solution for yy stresses in a multiple inclusion problem

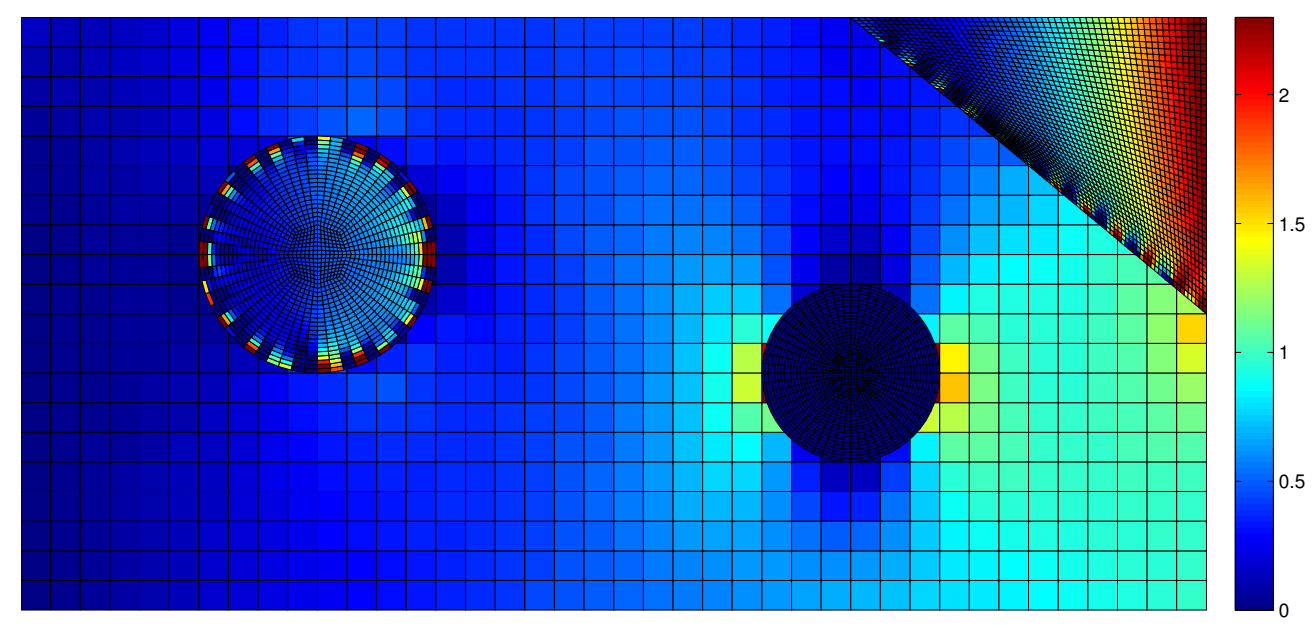

Figure 16: Standard mortar solution for yy stresses in a multiple inclusion problem 


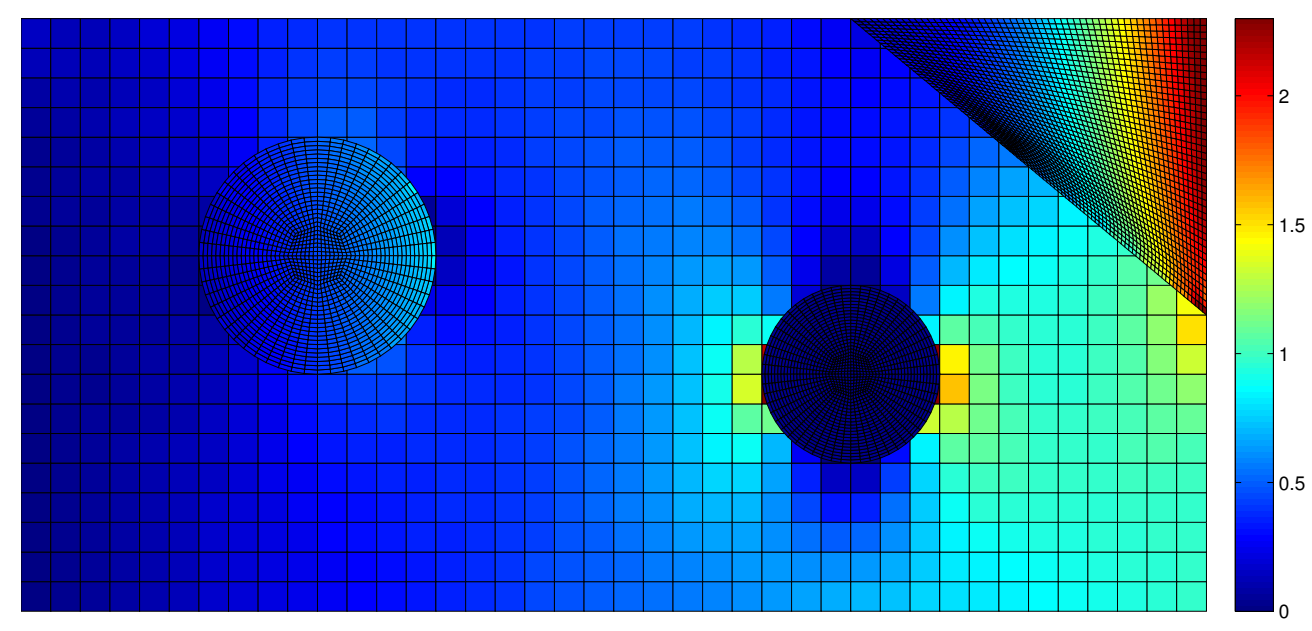

Figure 17: Penalty solution for yy stresses in a multiple inclusion problem

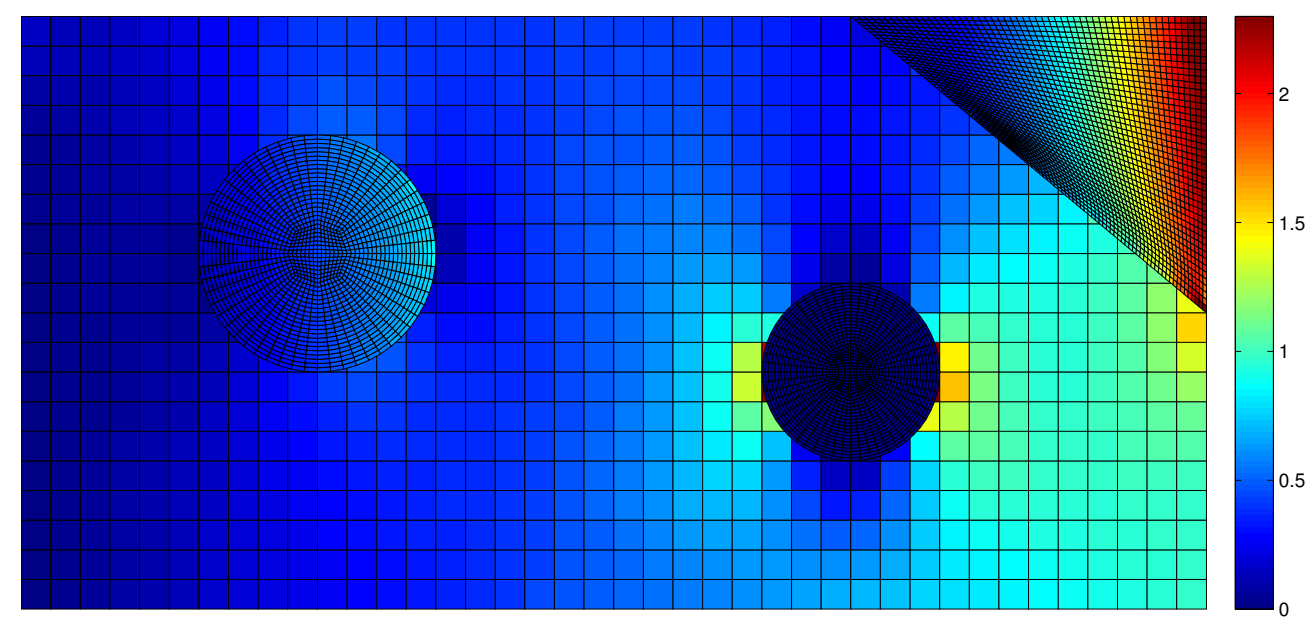

Figure 18: Weighted Nitsche solution for yy stresses in a multiple inclusion problem 

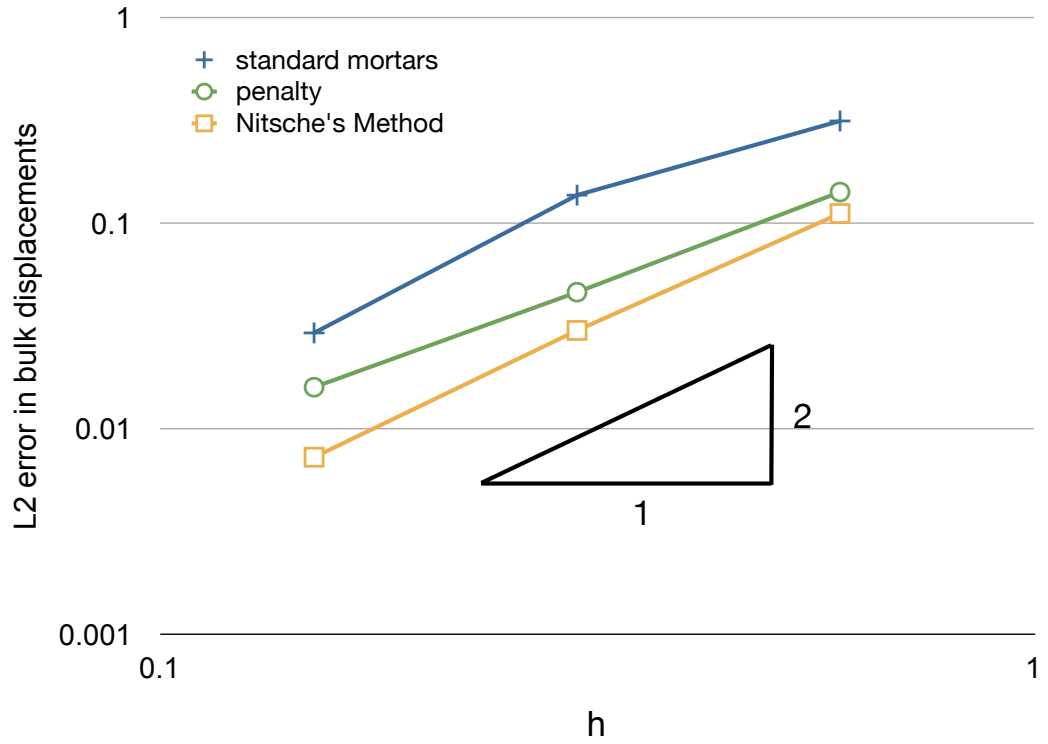

(a)

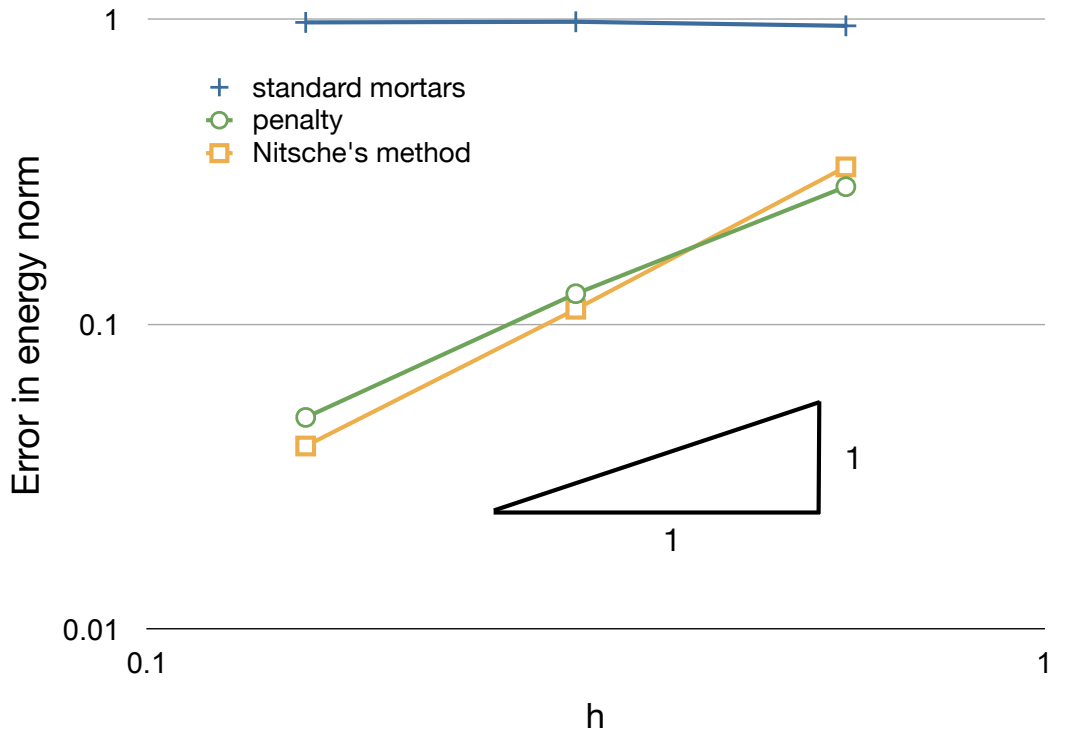

(b)

Figure 19: Convergence rates for standard mortars, a penalty method, and a weighted Nitsche's method 


\section{Conclusions}

It can be inferred that there are major advantages to using a Nitsche's method approach for embedded finite element constraints. With the correct biasing and choice of stabilization parameter, Nitsche's method never, to our knowledge, displays the same problems with mesh locking that standard mortar methods may. Additionally, there appear to be few extra challenges to extending Nitsche's method to three dimensions. For the case of embedded meshes, regardless of the constraint enforcement, the computational geometry of re-triangulating cut cells is much more complicated in three dimensions. Finally, the discrete expressions for the stress operators are not more complicated than the standard expressions used to calculate element stiffness.

One of the main drawbacks of Nitsche's method can be its sensitivity to the choice of stabilization parameter. Additionally, attempts to extend Nitsche's method as it was presented in this paper to non-linear materials have neither a precedent nor an obvious way forward, due to the specific form of the symmetry term. The obvious future work for this method is to overcome these problems and provide a robust extension to non-linear model equations.

The work of M. A. Puso was performed under the auspices of the U.S. Department of Energy by Lawrence Livermore National Laboratory under Contract DE-AC52-07NA27344.

\section{References}

[1] F P T Baaijens. A fictitious domain/mortar element method for fluid-structure interaction. International Journal for Numerical Methods in Engineering, 35:743-761, 2001.

[2] R Glowinski, T Pan, and Périaux. A fictitious domain method for dirichlet problems and applications. Computer Methods in Applied Mechanics and Engineering, 111:283-303, 1994.

[3] F Brezzi, J L Lions, and O Pironneau. Analysis of a Chimera method. Comptes Rendus De L'académie des Sciences - Serie I - Mathematics, 332(7):655-660, 2001.

[4] G Houzeaux and R. Codina. A chimera method based on dirichlet/neumann(robin) coupling for navier stokes. Computer Methods in Applied Mechanics and Engineering, 192:3343-3377, 2003.

[5] L Zhang, A Gerstenberger, XD Wang, and WK Liu. Immersed finite element method. Computer Methods in Applied Mechanics and Engineering, 193(21-22):2051-2067, 2004.

[6] W F Noh. A time-dependent, two space dimensional, coupled eulerian-lagrangian code. Methods in Computational Physics, 1964.

[7] H Ben Dhia and G Rateau. The arlequin method as a flexible engineering design tool. International Journal for Numerical Methods in Engineering, 62:1442-1462, 2005. 
[8] J D Sanders, J E Dolbow, and T A Laursen. On methods for stabilizing constraints over enriched interfaces in elasticity. International Journal for Numerical Methods in Engineering, 78:1009-1036, 2009.

[9] E Bechét, N Moës, and B Wohlmuth. A stable lagrange multiplier space for stiff interface conditions within the extended finite element method. International Journal for Numerical Methods in Engineering, 78:931-954, 2009.

[10] A Gerstenberger and W A Wall. An extended finite element method/lagrange multiplier based approach for fluid-structure interaction. Computer Methods in Applied Mechanics and Engineering, 197:1699-1714, 2008.

[11] J E Dolbow and I Harari. An efficient finite element method for embedded interface problems. International Journal for Numerical Methods in Engineering, 78:229-252, 2009.

[12] A Hansbo and P Hansbo. An unfitted finite element method, based on Nitsche's method, for elliptical interface problems. Computer Methods in Applied Mechanics and Engineering, 191:5537-5552, 2002.

[13] I. Babuška. The finite element method with penalty. Mathematics of Computation, 27:221-228, 1973.

[14] A J Lew and G C Buscaglia. A discontinuous-Galerkin based immersed boundary method. International Journal for Numerical Methods in Engineering, 76(4):427-454, 2008 .

[15] Rolf Stenberg. On some techniques for approximating boundary conditions in the finite element method. Journal of Computational and Applied Mathematics, 63:139-148, 1995.

[16] N Moës, J Dolbow, and T Belytschko. A finite element method for crack growth without remeshing. International Journal for Numerical Methods in Engineering, 46:131-150, 1999. 